\title{
Quantum mechanical reactive scattering for planar atom plus diatom systems. II. Accurate cross sections for $\mathrm{H}+\mathrm{H}_{2}{ }^{*}$
}

\author{
George C. Schatz ${ }^{\dagger}$ and Aron Kuppermann \\ Arthur Amos Noyes Laboratory of Chemical Physics, Division of Chemistry and Chemical Engineering, \\ California Institute of Technology, Pasadena, California 91125 \\ (Received 22 December 1975)
}

\begin{abstract}
The results of an accurate quantum mechanical treatment of the planar $\mathrm{H}+\mathrm{H}_{2}$ exchange reaction on a realistic potential energy surface are presented. Full vibration-rotation convergence was achieved in the calculations, and this, together with a large number of auxiliary convergence and invariance tests, indicates that the cross sections are accurate to $5 \%$ or better. The reactive differential cross sections are always backward peaked over the range of total energies from 0.3 to $0.65 \mathrm{eV}$. Nonreactive $j=0$ to $j^{\prime}=2$ cross sections are backward peaked at low energy $(0.4 \mathrm{eV})$ shifting to sidewards peaking for $E>0.5 \mathrm{eV}$. Quantum symmetry interference oscillations are very significant in the $j=0$ to $j^{\prime}=2$ para-to-para cross sections for $E \geq 0.6 \mathrm{eV}$. Reactive integral cross sections show two distinct kinds of energy dependence. At low energy $(<0.5 \mathrm{eV})$, barrier tunneling gives them a largely exponential energy dependence while above $0.5 \mathrm{eV}$ (the effective threshold energy) the cross sections vary nearly linearly. Comparison of collinear and coplanar transition probabilities indicates similar $1 D$ and 2D energy dependence but with a shift in energy from 1D to 2D due to bending motions in the transition state. An analysis of rotational distributions indicates surprisingly good correspondence with temperaturelike distributions. The results of a onevibration-approximation calculation are examined, and errors of as much as three orders of magnitude are found at some energies. Shapes of angular distributions are, however, accurately predicted by this approximate method. Additional analyses include comparisons with previous distorted wave and coupledchannel results, and calculations of thermal rate constants.
\end{abstract}

\section{INTRODUCTION}

A reaction of fundamental interest in the field of chemical dynamics is the $\mathrm{H}+\mathrm{H}_{2}$ hydrogen atom exchange reaction. This simplest of chemical reactions has been the subject of numerous dynamical studies by quasiclassical, ${ }^{1,2}$ semiclassical, ${ }^{3-5}$ and quantum mechanical $^{6-16}$ methods and has been the focal point for the development of many approximate reaction rate theories. ${ }^{17}$ In addition, this system provides the fundamental example for characterizing quantum effects in chemical reactions and determining their importance on experimental observables. For these reasons, the calculation of accurate quantum mechanical cross sections for $\mathrm{H}+\mathrm{H}_{2}$ is of great importance. Unfortunately, until recently there existed neither the proper methods for efficiently solving the Schrödinger equation for this system nor adequately powerful computers to handle the computations involved without the introduction of approximations of unknown accuracy.

In the preceding paper ${ }^{18}$ (hereafter referred to as I) we presented a method for accurately and efficiently solving the Schrödinger equation for reactive collisions of an atom with a diatomic molecule moving on a fixed plane. The planar motion restriction was introduced for computational simplicity only, with no fundamental limitations involved in applying a similar procedure to three-dimensional collisions as well. In this paper, we present the results of an application of this method to planar $\mathrm{H}+\mathrm{H}_{2}$ on a realistic potential energy surface. ${ }^{19}$ The results to be discussed include reactive and nonreactive transition probabilities, differential cross sections and integral cross sections, product rotational state distributions, and rate constants. In a preliminary communication, ${ }^{11}$ we examined the importance of closed vibrational channels in a vibrationrotation coupled-channel (i。e., close-coupling) ${ }^{20}$ expansion and found that the errors associated with an early truncation of the vibrational basis set expansion could be very serious in many cases although qualitative trends obtained with the truncated basis were often properly described. We will examine the one vibrational basis function approximation in somewhat greater detail in this paper, and will, in addition, compare our results with the approximate results of others in which different methods, types of approximations, and potential surfaces were used.

As pointed out in Paper I, the method we have developed for solving the Schrödinger equation for planar atom plus diatomic molecule collisions can be extended to 3D systems without significant conceptual changes, so an additional reason for undertaking the current calculations was to test the feasibility of the method in preparation for its application to $3 \mathrm{D}$ reactive systems. The calculations for the $3 \mathrm{D} \mathrm{H}+\mathrm{H}_{2}$ system have now been completed and are presented in detail in the following two papers. ${ }^{21}$ A preliminary communication of the results of this $3 \mathrm{D}$ work and its relationship with some of the coplanar results presented here has already been published. ${ }^{12}$

In Sec. II we describe the reactive scattering calculations, including convergence tests and computational considerations, and the representation of the potential energy surface. The results of the calculations are presented and discussed in Sec. III. Section IV contains a general summary and discussion. 


\section{QUANTUM MECHANICAL CALCULATIONS FOR PLANAR REACTIVE $\mathrm{H}+\mathrm{H}_{2}$}

\section{A. General description of the method}

The method used to solve the Schrödinger equation for planar reactive and nonreactive $\mathrm{H}+\mathrm{H}_{2}$ collisions has been extensively described in Paper $\mathrm{I}$. As outlined there, the procedure for obtaining the full set of primitive solutions to the partial-wave Schrödinger equation is divided into two stages. In the first one, a coupled-channel ${ }^{20}$ method is used to generate solutions to the Schrödinger equation in each of the three arrangement channel regions of internal configuration space. These solutions are then smoothly matched to one another in the second stage, and the resulting primitive solutions, which are everywhere smoothly continuous, are then linearly combined to yield the appropriate reactance and scattering matrix solutions. This procedure is then repeated for a sufficient number of partial waves to obtain converged reactive, inelastic, and (if desired) elastic cross sections. The potential energy surface used in all the calculations was the semiempirical ground electronic state $\mathrm{H}_{3}$ surface of Porter and Karplus ${ }^{19}$ (all coupling to excited electronic surfaces being neglected). In solving the Schrödinger equation for these reactive collision systems, great care must be exercised to insure adequate invariance of the results with respect to a change in (a) the number of vibration-rotation basis functions used, (b) the refer- ence potential $V_{\text {rer }}$ used to generate these functions, (c) the representation of the potential surface (see Sec. II. B), and (d) the nature of the functions used to represent the wavefunction on the matching surface (i.e., the "matching surface basis functions" of Paper I).

As discussed in Paper $I$, a number of symmetry properties inherent in $\mathrm{H}+\mathrm{H}_{2}$ and similar systems may be utilized to reduce the computation time involved in doing these calculations. Most significant in this respect are (a) cyclic permutational symmetry of the three-atom system which allows one to consider only one arrangement channel region in doing all calculations, and (b) two-atom permutational symmetry, which allows one to decouple the even and odd rotational states throughout most of the calculation. These same symmetry properties allow us to reduce the number of different distinguishable atom scattering amplitudes between a given initial vibration-rotation state of the reagent $\mathrm{H}_{2}$ and a given final state of the product $\mathrm{H}_{2}$ to just two: one reactive and one nonreactive amplitude. We shall denote the reagent diatomic states by the vibration-rotation quantum numbers $v j$ and the product ones by $v^{\prime} j^{\prime}$. Distinguishable-atom reactive transitions will be designated by the superscript $R$, nonreactive ones by $N$, and (indistinguishable) antisymmetrized ones by $A$. In this notation, the relation between the antisymmetrized differential cross sections and the distinguishable-atom dimensionless scattering amplitudes [Eqs. $(6,5)$ of Paper I] is

$$
\sigma_{v f-v^{\prime} j^{\prime}}^{A}= \begin{cases}\frac{1}{k_{v j}}\left|f_{v j-v^{\prime} j^{\prime}}^{N}-f_{v j-v^{\prime} j^{\prime}}^{R}\right|^{2} \quad\left(j, j^{\prime} \text { even, para - para }\right) \\ \frac{3}{k_{v j}}\left|f_{v j \rightarrow v^{\prime} j^{\prime}}^{R}\right|^{2}=3 \sigma_{v j \rightarrow v^{\prime} j^{\prime}}^{R} & \left(j \text { even, } j^{\prime} \text { odd, para } \rightarrow \text { ortho }\right) \\ \frac{1}{k_{v j}}\left|f_{v j-v^{\prime} j^{\prime}}^{R}\right|^{2}=\sigma_{v j-v^{\prime} j^{\prime}}^{R} & \left(j \text { odd, } j^{\prime} \text { even, ortho - para }\right) \\ \frac{1}{k_{v j}}\left(\left|f_{v j \rightarrow v^{\prime} j^{\prime}}^{N}+f_{v j \rightarrow v^{\prime} j^{\prime}}^{R}\right|^{2}+2\left|f_{v f \rightarrow v^{\prime} j^{\prime}}^{R}\right|^{2}\right) \quad\left(j, j^{\prime} \text { odd, ortho } \rightarrow \text { ortho }\right),\end{cases}
$$

where $k_{v j}$ is the (unscaled) wave number $\left(\bar{k}_{p j}\right.$ of Paper I), and $f_{v f \rightarrow v^{\prime} j^{\prime}}^{N}$ and $f_{v f \rightarrow v^{*} j^{\prime}}^{R}$ were denoted by $\hat{f}_{\lambda v f^{\prime}}^{\lambda v^{\prime}}$ and $\hat{f}_{\lambda v f}^{v v^{*} f^{\prime}}$, respectively, in Paper I. For planar systems, the diatom rotational quantum number $j$ is an algebraic integer and may be either positive, negative, or zero. For $j \neq 0$, the two states $j$ and $-j$ are degenerate and said to have different polarizations. Differential cross sections which have been summed over final rotational polarizations and averaged over initial ones will be indicated by the symbol $\bar{\sigma}_{v f \rightarrow v^{\prime} f^{\prime}}$ and the corresponding integral cross sections by $\bar{Q}_{v j-v^{\prime} j^{\prime}}$. For example, the integral cross section $\bar{Q}_{01,02}^{R}$ is given by

$$
\bar{Q}_{01 \rightarrow 02}^{R}=\frac{1}{2}\left(Q_{01-02}^{R}+Q_{01-0,-2}^{R}+Q_{0,-1-02}^{R}+Q_{0,-1-0,-2}^{R}\right) \text { 。 }
$$

In Sec. V. B of Paper I we found that the symmetry of the Hamiltonian with respect to reflection through the triatom plane leads to the following relations between cross sections within the same rotational manifolds (valid for $R, N$, or $A$ transitions):

$$
\sigma_{v f \rightarrow v^{\prime} f^{\prime}}(\theta)=\sigma_{v_{r}-f \rightarrow v^{\prime},-f^{\circ}}(2 \pi-\theta)
$$

and

$$
Q_{v f-v^{\prime} f^{\prime}}=Q_{v_{1}-f \rightarrow v^{\prime},-f^{\prime}}
$$

As defined in Paper I, the scattering angle $\theta$ is the angle between the directions of motion of the final and initial $\mathrm{H}$ atoms in the center of mass system and spans the range $0 \leq \theta \leq 2 \pi$. For reactive differential cross sections, the more customary angle to use is the angle $\theta_{R}$ of the direction of the product $\mathrm{H}_{2}$ with respect to the direction of the incident $H$, and is related to $\theta$ by

$$
\theta_{R}=\theta+\pi \quad(\bmod 2 \pi) .
$$

Therefore, the backward reactive scattering direction corresponds to $\theta_{R}=\pi$ and $\theta=0$. 


\section{B. Representation of the potential energy surface}

In setting up the coupled differential equations which must be solved in each arrangement channel region $\lambda=\alpha, \beta, \gamma$, the potential energy surface $V^{\lambda}\left(r_{\lambda}, R_{\lambda}, \gamma_{\lambda}\right)$ is expanded (see definitions in Paper I) in a cosine Fourier series of the angle $\gamma_{\lambda}[\mathrm{Eq},(3,8 \mathrm{a})$ of Paper I]:

$$
V^{\lambda}\left(r_{\lambda}, R_{\lambda}, \gamma_{\lambda}\right)=\sum_{k=0}^{\infty} V_{k}^{\lambda}\left(r_{\lambda}, R_{\lambda}\right) \cos k \gamma_{\lambda} \text {. }
$$

In the case of the $\mathrm{H}+\mathrm{H}_{2}$ reaction, $V^{\lambda}\left(r_{\lambda}, R_{\lambda}, \gamma_{\lambda}\right)$ is symmetric about $\gamma_{\lambda}=\pi / 2$ and $3 \pi / 2$ [Eq. (6.1) of Paper I], so only even $k$ terms need be included in Eq. (2.6). Once the coefficients $V_{k}^{\lambda}\left(r_{\lambda}, R_{\lambda}\right)$ are determined, the rotational coupling in the Schrödinger equation can be analytically evaluated [as in Eq. $(3,9)$ of Paper I]; this greatly facilitates the determination of the potential matrix elements needed in the integration procedure. Unfortunately, in general, the $V_{k}^{\lambda}\left(r_{\lambda}, R_{\lambda}\right)$ must be calculated numerically from the relation

$$
V_{k}^{\lambda}\left(r_{\lambda}, R_{\lambda}\right)=\frac{2}{\left(1+\delta_{k 0}\right) \pi} \int_{0}^{\pi} \cos k \gamma_{\lambda} V^{\lambda}\left(r_{\lambda}, R_{\lambda}, \gamma_{\lambda}\right) d \gamma_{\lambda},
$$

and the effort involved in computing this integral negates the advantage of using an expression which is analytical in $\gamma_{\lambda}$ such as Eq. (2.6). However, for the Porter-Karplus potential surface ${ }^{19}$ (and for many others as well), in the regions of internal configuration space sampled in the calculation, the expansion Eq. (2.6) converges very rapidly (after only three or four terms). We can then redefine the $V_{k}^{\lambda}$ by requiring that, instead of satisfying Eq。(2.7), they force Eq. (2.6), with a finite number $n$ of even terms, to be satisfied exactly at $n$ values of $\gamma_{\lambda}$. For example, if three terms are included, then we can find $V_{0}^{\lambda}, V_{2}^{\lambda}$, and $V_{4}^{\lambda}$ by solving the three algebraic equations obtained when Eq. (2.6), truncated after three even terms, is evaluated at $\gamma=0$, $\pi / 4$, and $\pi / 2$. The result is

$$
\left(\begin{array}{c}
V_{0}^{\lambda}\left(r_{\lambda}, R_{\lambda}\right) \\
V_{2}^{\lambda}\left(r_{\lambda}, R_{\lambda}\right) \\
V_{4}^{\lambda}\left(r_{\lambda}, R_{\lambda}\right)
\end{array}\right)=\left(\begin{array}{ccc}
\frac{1}{4} & \frac{1}{2} & \frac{1}{4} \\
\frac{1}{2} & 0 & -\frac{1}{2} \\
\frac{1}{4} & -\frac{1}{2} & \frac{1}{4}
\end{array}\right)\left(\begin{array}{c}
V^{\lambda}\left(r_{\lambda}, R_{\lambda}, \gamma_{\lambda}=0\right) \\
V^{\lambda}\left(r_{\lambda}, R_{\lambda}, \gamma_{\lambda}=\pi / 4\right) \\
V^{\lambda}\left(r_{\lambda}, R_{\lambda}, \gamma_{\lambda}=\pi / 2\right)
\end{array}\right) .
$$

For a small number of terms in the potential function expansion, the above interpolative procedure yields a representation of the full potential function $V^{\lambda}\left(r_{\lambda}, R_{\lambda}\right.$, $\gamma_{\lambda}$ ) which is computationally more efficient but has about the same accuracy as the one generated using Eq. (2.7)。 Of course, the goodness of this procedure depends very significantly on the nature of the potential energy surface being considered, but for the PorterKarplus $\mathrm{H}_{3}$ surface, it allows an adequate representation of the potential while requiring an exact evaluation of $V^{\lambda}$ at only three or four values of $\gamma_{\lambda}$ [and the use of Eq. (2.6) for all others]. In Fig. 2, of Paper $I^{18}$ we depicted equipotential contours of the potential energy surface at $\gamma_{\lambda}=0, \pi / 4$, and $\pi / 2$, the values required in the evaluation of Eq. (2.8).

\section{Convergence and accuracy tests}

It is of crucial importance in coupled-channel calculations to establish that the resulting reaction probabilities and cross sections have converged adequateIy. Indeed, we shall see later that premature truncation of the vibration-rotation basis set expansion can result in errors in the final integral cross sections by several orders of magnitude, even though other tests, such as conservation of flux, may be approximately satisfied. Furthermore, many approximation quantum methods involve various kinds of truncations and/or other approximations, and it is highly desirable to obtain fully converged results which are of sufficient accuracy to assess the validity of those methods.

The most obvious criteria which must be satisfied by the results of an accurate quantum calculation are conservation of flux and time reversal invariance. These two principles require that the scattering matrix
$\mathbf{S}_{\boldsymbol{J}}$ be unitary and symmetric for each total angular momentum quantum number $J$ and therefore that the corresponding probability matrix $\mathbf{P}_{J}$ [defined by Eq. (5.20) of Paper I] be symmetric and that the sum of the elements of each of its rows (or columns) should equal unity. These criteria are necessary but not sufficient to insure accurate results.

In the results to be discussed in detail in Sec. III, we consider a range of total energies $E$ from 0.30 to $0.75 \mathrm{eV}$ (translational energies relative to the $v=0$, $j=0$ reagent $\mathrm{H}_{2}$ state of $0.03-0.48 \mathrm{eV}$ ). Flux conservation and microscopic reversibility were checked in each calculation, and for $E \leq 0.60 \mathrm{eV}$, deviations from flux conservation were never worse than $1 \%$ and from symmetry less than about $5 \%$ (for nonnegligible transition probabilities)。 For $0.60 \mathrm{eV}<E \leq 0.75 \mathrm{eV}$, deviations from flux conservation were less than $3 \%$ and from symmetry less than $10 \%$. In order to insure satisfactory convergence (better than $5 \%$ ) in the calculation, vibration-rotation basis sets including 40-60 terms (channels) were required. For energies less than 0.50 $\mathrm{eV}$, a 40 channel basis consisting of 5 vibrational wavefunctions combined with $10,10,8,6$, and 6 rotational wavefunctions for $v=0,1,2,3$, and 4 , respectively, were used in general. In the $0.50-0.60 \mathrm{eV}$ range, a 48 channel basis set of 4 vibrations and 12 rotations per vibration was adequate, while for energies above $0.60 \mathrm{eV}$, a 60 channel basis of 5 vibrations and 12 rotations per vibration was used. Typical probability matrices from these calculations (for a 48 channel calculation at $0.55 \mathrm{eV}$ ) are given in Table I. Both the reactive and nonreactive transition probability matrices are highly symmetric, and the sums of the probabilities in each row or column differs from unity by a 
TABLE I. Nonreactive (N) and reactive (R) probability matrices for $E=0.55 \mathrm{eV}, J=2 .{ }^{\mathrm{a}}$

\begin{tabular}{llllllll}
\hline \hline$(v j)$ & $\left(v^{\prime} j^{\prime}\right)=(00)$ & $(01)$ & $(0,-1)$ & $(02)$ & $(0,-2)$ & $(03)$ & $(0,-3)$ \\
& & & & $\mathrm{N}$ & & \\
$(00)$ & 0.0186 & $0.263(-8)$ & $0.113(-6)$ & 0.404 & 0.276 & $0.370(-7)$ & $0.248(-7)$ \\
$(01)$ & $0.287(-7)$ & 0.0134 & 0.477 & $0.689(-7)$ & $0.104(-7)$ & 0.199 & 0.0629 \\
$(0,-1)$ & $0.337(-7)$ & 0.477 & 0.0899 & $0.897(-7)$ & $0.112(-7)$ & 0.129 & 0.140 \\
$(02)$ & 0.406 & $0.204(-7)$ & $0.228(-7)$ & 0.240 & 0.172 & $0.289(-7)$ & $0.108(-7)$ \\
$(0,-2)$ & 0.277 & $0.530(-8)$ & $0.298(-7)$ & 0.171 & 0.474 & $0.287(-8)$ & $0.496(-8)$ \\
$(03)$ & $0.633(-8)$ & 0.199 & 0.129 & $0.126(-8)$ & $0.343(-8)$ & 0.636 & $0.651(-2)$ \\
$(0,-3)$ & $0.527(-8)$ & 0.0624 & 0.140 & $0.327(-8)$ & $0.468(-8)$ & $0.653(-2)$ & 0.785 \\
& & & & & & & \\
$(00)$ & 0.0436 & 0.0380 & 0.0265 & 0.0167 & $0.0803(-1)$ & $0.0347(-1)$ & $0.911(-3)$ \\
$(01)$ & 0.0380 & 0.0357 & 0.0219 & 0.0178 & $0.0567(-1)$ & $0.0378(-1)$ & $0.621(-3)$ \\
$(0,-1)$ & 0.0261 & 0.0220 & 0.0167 & $0.0980(-1)$ & $0.0502(-1)$ & $0.0181(-1)$ & $0.664(-3)$ \\
$(02)$ & 0.0174 & 0.0175 & $0.0961(-1)$ & $0.0857(-1)$ & $0.0246(-1)$ & $0.0211(-1)$ & $0.232(-3)$ \\
$(0,-2)$ & $0.0774(-1)$ & $0.0588(-1)$ & $0.0518(-1)$ & $0.0232(-2)$ & $0.0180(-1)$ & $0.412(-3)$ & $0.243(-3)$ \\
$(03)$ & $0.0356(-1)$ & $0.0373(-1)$ & $0.0184(-1)$ & $0.0206(-1)$ & $0.412(-3)$ & $0.500(-3)$ & $0.407(-4)$ \\
$(0,-3)$ & $0.879(-3)$ & $0.619(-3)$ & $0.655(-3)$ & $0.237(-3)$ & $0.234(-3)$ & $0.398(-4)$ & $0.386(-4)$ \\
Sums ${ }^{\mathrm{b}}$ & 1.0014 & 0.9997 & 1.0003 & 0.9980 & 1.0001 & 0.9999 & 1.0005 \\
\hline \hline
\end{tabular}

${ }^{a}$ Not all allowed transitions are shown. Numbers in parentheses indicate powers of 10 by which numbers preceding them should be multiplied.

${ }^{b}$ Sum of probabilities from a given initial state over all possible final states and arrangement channels.

very small amount in every case. In Table II we examine the convergence behavior of the transition probabilities both as the number of vibrations per rotation is increased and as the number of rotations per vibration is increased (all at $0.6 \mathrm{eV}$ ). In Part $\mathrm{A}$ of that table we see that the results change by less than $5 \%$ in going from 12 to 14 rotations per vibration and by somewhat larger amounts in going from 10 to 14 . With fewer than 10 rotations, errors of $10 \%$ to nearly $100 \%$ are observed in certain transition probabilities. When vibrational convergence is examined (Part B) of Table II), we find that $2 \%$ convergence is attained with 4 vibrations and that the use of fewer than that number can lead to errors as large as 50\% along with poor flux conservation.

Another important accuracy test in these calculations is the invariance of the results to changes in the character of the vibration-rotation basis set. There are two important ways to test this. First, one should be able to change the number of rotations per vibration or the number of vibrations per rotation without changing the results as long as convergence has been attained. Second, the results should be independent of the reference potential $V_{\text {ref }}\left(r_{\lambda}, R_{\lambda}\right)$ [Eq. (3.36) of Paper I] which serves to define the vibrational basis functions as long as $V_{\text {ref }}$ becomes equal to the correct diatomic potential $v\left(r_{\lambda}\right)$ in the limit $R_{\lambda} \rightarrow \infty$. In Table III we present the results of these two kinds of tests. The first column tabulates representative nonreactive and reactive transition probabilities for $E=0.50 \mathrm{eV}, J=0$ calculations with a 48 channel basis (4 vibrations, 12 rotations/vibration) and a reference potential $V_{\text {ref }}$ $=V\left(\gamma_{\lambda}, R_{\lambda}, \gamma_{\lambda}=0\right)$ (the one actually used in most of the calculations). In the second column we give the corresponding probabilities obtained when the reference potential $V_{\text {ref }}=V_{0}^{\lambda}\left(r_{\lambda}, R_{\lambda}\right)$ is used [Eqs. $(2.6)$ and (2.8)]. Finally, in the last column we give the transition prob- abilities obtained with the $V_{0}^{\lambda}\left(r_{\lambda}, R_{\lambda}\right)$ reference potential and a 50 channel basis ( 5 vibrations, $12,12,10,8$, and 8 rotations in $v=0,1,2,3$, and 4 , respectively). The deviations between the corresponding probabilities is less than $5 \%$. This result is typical of the accuracy for energies $E \leq 0.60 \mathrm{eV}$. Somewhat larger changes are found for $0.6 \mathrm{eV}<E \leq 0.75 \mathrm{eV}$, but usually less than $10 \%$.

Two additional accuracy tests are (a) convergence of the results with respect to the number of terms in the expansion of the potential [Eq. (2.6)], and (b) invariance

TABLE II. Rotational and vibrational convergence of coplanar transition probabilities at $E=0.60 \mathrm{eV}, J=1$.

\begin{tabular}{rcccccc}
\hline \hline$N$ & $U^{\mathrm{a}}$ & $P_{00 \rightarrow 02}^{N}$ & $P_{01-0-1}^{N}$ & $P_{00-00}^{R}$ & $P_{00-01}^{R}$ & $P_{01 \rightarrow 0-1}^{R}$ \\
\hline A. & $\begin{array}{l}\text { Rotational convergence (with } \\
\text { per vibration) }\end{array}$ \\
6 & 1.035 & 0.293 & 0.435 & 0.0738 & 0.0667 & 0.0356 \\
8 & 1.020 & 0.276 & 0.339 & 0.0743 & 0.0525 & 0.0380 \\
10 & 1.010 & 0.202 & 0.257 & 0.0840 & 0.0706 & 0.0416 \\
12 & 1.004 & 0.194 & 0.230 & 0.0829 & 0.0645 & 0.0396 \\
14 & 1.002 & 0.189 & 0.221 & 0.0821 & 0.0673 & 0.0397
\end{tabular}

B. Vibrational convergence (with $M$ vibrations and 12 rotations per vibration)

\begin{tabular}{lllllll}
$1^{\text {b }}$ & 1.003 & 0.259 & 0.260 & 0.0404 & 0.0372 & 0.0295 \\
2 & 1.063 & 0.161 & 0.204 & 0.0895 & 0.0738 & 0.0477 \\
3 & 1.063 & 0.238 & 0.315 & 0.0749 & 0.0578 & 0.0329 \\
4 & 1.004 & 0.194 & 0.230 & 0.0829 & 0.0645 & 0.0396 \\
5 & 1.007 & 0.195 & 0.233 & 0.0832 & 0.0646 & 0.0396 \\
\hline \hline
\end{tabular}

${ }^{a} U$ indicates the sum of all transition probabilities from a specific $v j$ state which differs by the largest amount from unity and hence is a conservative measure of deviations from flux conservation.

${ }^{b_{T}}$ The one vibration results were calculated according to the procedure outlined in Sec. II.D. 
TABLE III. Nonreactive and reactive transition probabilities for $E=0.50 \mathrm{eV}, J=0$.

\begin{tabular}{llll}
\hline & $N=48, V_{\text {coll }}{ }^{\mathrm{a}}$ & $N=48, V_{0}{ }^{\mathrm{b}}$ & $N=50, V_{0}{ }^{\mathrm{c}}$ \\
\hline$P_{00 \rightarrow 00}^{N}$ & 0.180 & 0.180 & 0.183 \\
$P_{00 \rightarrow 0 \pm 2}^{N}$ & 0.383 & 0.383 & 0.383 \\
$P_{0 \pm 1 \rightarrow 0 \pm 1}^{N}$ & 0.207 & 0.207 & 0.211 \\
$P_{0 \pm 1 \rightarrow 0 \mp 1}^{N}$ & 0.583 & 0.582 & 0.580 \\
$P_{00 \rightarrow 00}^{R}$ & $0.787 \times 10^{-2}$ & $0.787 \times 10^{-2}$ & $0.755 \times 10^{-2}$ \\
$P_{00 \rightarrow 0 \pm 1}^{R}$ & $0.580 \times 10^{-2}$ & $0.578 \times 10^{-2}$ & $0.557 \times 10^{-2}$ \\
$P_{0 \pm 1-0 \pm 1}^{R}$ & $0.422 \times 10^{-2}$ & $0.419 \times 10^{-2}$ & $0.404 \times 10^{-2}$ \\
$P_{0 \pm 1 \rightarrow 0 \mp 1}^{R}$ & $0.413 \times 10^{-2}$ & $0.410 \times 10^{-2}$ & $0.395 \times 10^{-2}$ \\
\hline \hline
\end{tabular}

48 channels $\left(v=0-3 ; 12\right.$ rotations/vibration), $V_{\text {rer }}=V_{\text {coll }}$ $=V\left(r_{\lambda}, R_{\lambda}, \gamma_{\lambda}=0\right)$.

b 48 channels $\left(v=0-3 ; 12\right.$ rotations/vibration), $V_{\text {ref }}=V_{0}^{\lambda}\left(r_{\lambda}, R_{\lambda}\right)$.

'50 channels $(v=0-4 ; 12,12,10,8,8$ vibrations for $v=0,1$,

$2,3,4$, respectively), $V_{\text {ref }}=V_{0}^{\lambda}\left(r_{\lambda}, R_{\lambda}\right)$.

of the results with respect to a change in the matching surface basis functions $B^{\nu \lambda}$ [Eq. (4.7) of Paper I]. We find that the reaction probabilities change by less than $5 \%$ in going from three to four terms in Eq. (2.6) [with the coefficients calculated as described before Eq. (2.8)] and virtually not at all in going from four to five terms. All calculations reported in this paper were done with three terms in Eq. (2.6) and using Eq. (2.8) to calculate $V_{0}, V_{2}$, and $V_{4}$. The effects of completeness of the expansion of the wavefunction on the matching surface were studied in two ways. First, several different choices of matching surface basis functions $B^{\nu \lambda}$ were used [different sine and cosine combinations (see Paper I) and Legendre polynomials] and invariance of the results to within $2 \%$ was found. Best unitarity of $\mathbf{S}_{J}$ was obtained with the basis set (c) of Sec. IV. A of Paper I, and this choice was used in all further calculations. Second, the degree of completeness of the matching surface basis functions $B^{\nu \lambda}$ determines the degree of orthogonality of the matrix $\mathbf{s}_{\nu \lambda}^{J}$ of Eq. (4.32) of Paper I which transforms the solution in $\nu$ coordinates to that in coordinates $\lambda$. This property of $\mathbf{s}_{\nu \lambda}^{J}$ determines the unitarity property of the scattering matrix $\mathbf{S}_{J}$ to a certain extent but it is also necessary if the nonreactive transition probabilities between even and odd rotational states are to vanish as required by the symmetry of the $\mathrm{H}_{3}$ system [see Eq. (6.4) of Paper I]. Examples of the effects of a nonorthogonal $\mathbf{s}_{v \lambda}^{J}$ are seen in Table I, where the nonreactive ortho to para transition probabilities typically have magnitudes of $10^{-7}$ rather than $10^{-25}$, which is more typically the case $\mathrm{e}^{22}$ when the orthogonality is built in through the use of Eq. $(4.30)$ of Paper I. In that equation, the matrix $\overline{\mathbf{s}}_{v \lambda}^{J}$ (the complex counterpart of $\left.\mathbf{s}_{\nu \lambda}^{J}\right)$ is related to a real symmetric matrix $\Delta^{\nu \lambda}$ via

$$
\overline{\mathbf{s}}_{\nu \lambda}^{J}=\exp \left(i J \Delta^{\nu \lambda}\right) 。
$$

This expression is inherently unitary even when a truncated basis is used to calculate $\Delta^{\nu \lambda}$. It should be apparent that this error is of negligible importance for the example given in Table $I$, but as $J$ increases, the deviations from orthogonality of $\mathbf{s}_{\nu \lambda}^{J}$ also increase. Fortunately, the reaction probabilities decrease rapidly as this happens and since a nonorthogonal matching procedure has no effect on inelastic transition probabilities in the absence of reaction, ${ }^{23}$ the problem with completeness of the matching surface functions disappears at higher $J$. No artificial orthogonalization procedures were introduced in the calculation (such as were used by Saxon and Light ${ }^{8}$ ), and therefore unitarity of $\mathbf{S}_{J}$ and zeroness of the even-to-odd reactive transition probabilities are tests of the completeness of $B^{\nu \lambda}$.

We conclude this section by quoting some computation times for these calculations. Both the integration and matching times vary roughly as $N^{3}$ for $N>20$, where $N$ is the number of channels. For 48 channel calculations using an IBM 370-158 computer, about 22 min of computation time per partial wave $J$ was required, of which $17 \mathrm{~min}$ was spent in the integration of the coupled equations and the rest in the matching and asymptotic analysis. About 13 partial waves $(J=0-12)$ were required for convergence of the reactive cross sections and 30 partial waves $(J=0-29)$ for convergence of the inelastic nonreactive cross sections at energies near $E=0.50 \mathrm{eV}$.

\section{The one-vibrational-basis-function approximation (OVA)}

An often used ${ }^{8-10}$ (but seldom justified) approximation in quantum calculations has been the neglect of closed vibrational channels in the vibration-rotation coupledchannel expansion. For $\mathrm{H}+\mathrm{H}_{2}$ at low energies, only the ground vibrational level is open, so this approximation involves the use of only one vibrational basis function plus a complete set of rotational functions for that vibration. The main reason for using this approximation is the large reduction in computation time (by 1-2 orders of magnitude for $\mathrm{H}+\mathrm{H}_{2}$ ) compared to a vibrationally converged calculation. One of the objectives of this paper is to examine the accuracy of this approximation by comparing the results of its application with the fully converged ones.

The procedure that we have used to perform these one-vibration-approximation (OVA) calculations is almost identical to the fully converged one outlined in Paper I. The following modifications are, however, needed:

(a) The overlap matrix $\mathbf{S}_{i}^{\lambda}$ between the vibration-rotation basis sets in subregions $i$ and $i+1$ [Eq. (3.42) of Paper I] is orthogonalized according to the Schmidt procedure. $^{24}$ This is required because otherwise the strongly nonorthogonal overlap matrix associated with this severe truncation of the vibrational expansion results in an excessive lack of flux conservation.

(b) The effective potential matrix in the strong interaction region (and analogously in the matching region) is modified to [see Eq. $(3,48)$ of Paper I]

$$
\begin{aligned}
& \left(\overline{\mathbf{U}}_{J}^{\lambda(s)}\right)_{v_{\lambda} J_{\lambda}^{\prime} J_{\lambda}^{\prime}}^{J^{\prime}}=\left\langle v_{\lambda}\right| \rho_{\lambda}^{2}\left[\frac{2 \mu}{\hbar^{2}}\left[V_{j \lambda J \lambda}^{\lambda}-\delta_{j_{\lambda} j_{\lambda}^{\prime}}\left(V_{\mathrm{ref}}^{\lambda}+E-\epsilon_{v_{\lambda}}^{\lambda(s)}\right)\right]\right. \\
& \left.\quad+\left(\frac{j_{\lambda}^{2}-\frac{1}{4}}{\left(r_{\lambda_{0}}-\rho_{\lambda} \cos \varphi_{\lambda}\right)^{2}}+\frac{\left(J-j_{\lambda}\right)^{2}-\frac{1}{4}}{\left(R_{\lambda_{0}}-\rho_{\lambda} \sin \varphi_{\lambda}\right)^{2}}-\frac{1}{4 \rho_{\lambda}^{2}}\right)\right]\left|v_{\lambda}^{\prime}\right\rangle,
\end{aligned}
$$




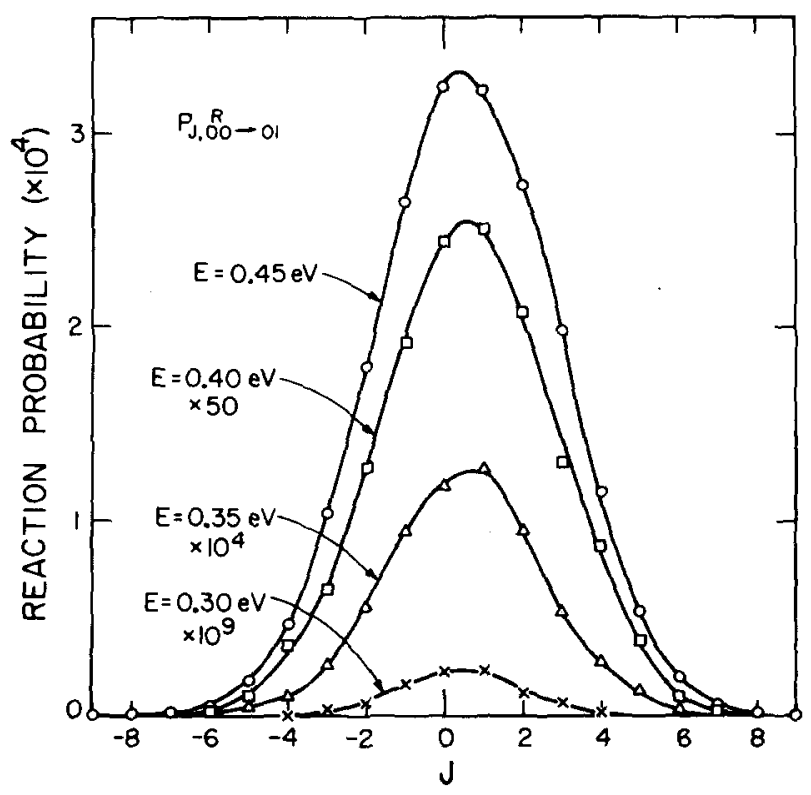

FIG. 1. Converged coplanar reaction probability $P_{J, 00-01}^{R}$ (for the $v=0, j=0 \rightarrow v^{\prime}=0, j^{\prime}=1$ transition) as a function of the total angular momentum quantum number $J$ for $E=0.30 \mathrm{eV}$ (crosses), $0.35 \mathrm{eV}$ (triangles), $0.40 \mathrm{eV}$ (squares), and $0.45 \mathrm{eV}$ (circles)。 Smooth curves have been drawn through the points.

where the only allowed values of $v_{\lambda}^{\prime}$ and $v_{\lambda}$ are zero. For a complete vibration-rotation basis set expansion, this expression is identical to that in Eq. (3.48) of Paper I, but in the OVA they differ, the above expression being the more consistent one. ${ }^{25}$

Even with these modifications, there are still many ambiguities in the application of this procedure. The most serious of these is the lack of invariance of the results to our choice of $V_{\text {ref }}\left(r_{\lambda}, R_{\lambda}\right)$. In Sec. III we shall examine results for $V_{\text {ref }}=V\left(r_{\lambda}, R_{\lambda}, \gamma_{\lambda}=0\right)$ and $V_{\text {ref }}=V_{0}^{\lambda}\left(r_{\lambda}, R_{\lambda}\right)$, with the hope that the range of results provided by these two calculations is representative of what can generally be obtained in this approximation.

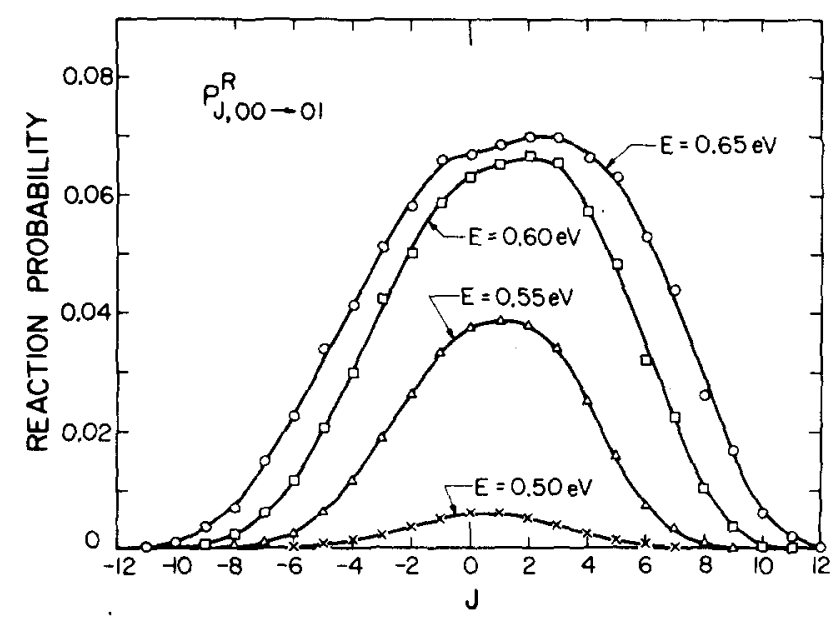

FIG. 2. Reaction probability $P_{J, 00-01}^{R}$ as a function of the total angular momentum quantum number $J$ analogous to $F$ ig. 1 but at total energies of $0.50 \mathrm{eV}$ (crosses), $0.55 \mathrm{eV}$ (triangles), $0.60 \mathrm{eV}$ (squares), and $0.65 \mathrm{eV}$ (circles).

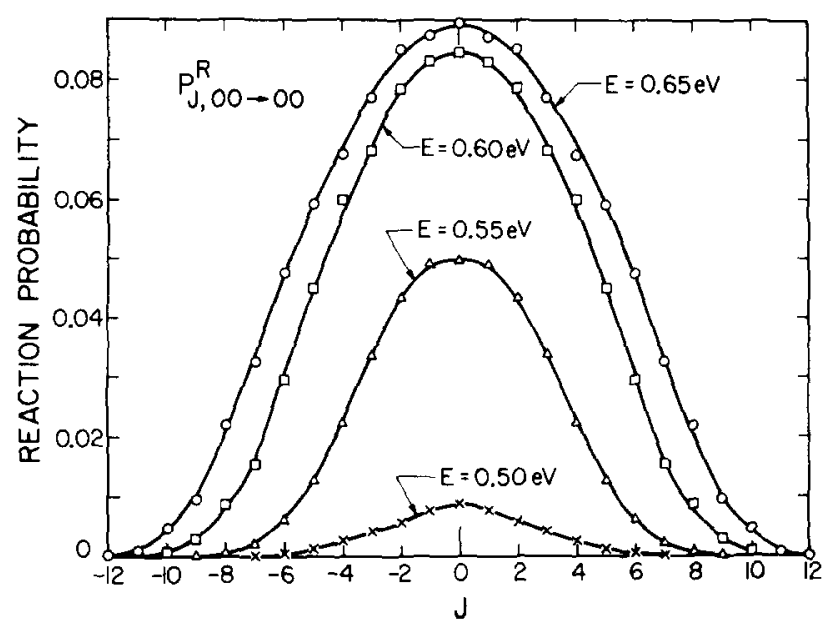

FIG. 3. Converged coplanar reaction probability $P_{\boldsymbol{J}, 00 \rightarrow 00}^{R}$ as a function of the total angular momentum quantum number $J$. Symbols correspond to same values of energy $E$ as in Fig. 2.

\section{RESULTS FOR COPLANAR $\mathrm{H}+\mathrm{H}_{2}$}

\section{A. Transition probabilities}

In this section we shall examine the $J$ dependence of the distinguishable-atom reactive and inelastic nonreactive transition probabilities. The reactive probabilities $P_{y, 00 \rightarrow 01}^{R}$ (for the $v=0, j=0 \rightarrow v^{\prime}=0, j^{\prime}=1$ transition) are plotted in Figs。 1 and 2 for several energies as a function of $J$. The probabilities for negative $J$ are obtained from those for positive $J$ through the use of the relation [resulting from Eqs. (5.20) and (5.32) of Paper I and valid for $R$ and $N$ probabilities]

$$
P_{J, v f \rightarrow v^{*} f^{*}}=P_{-J, v,-f-v^{*},-j^{*}} \text {. }
$$

Figures 1 and 2 indicate that $P_{J_{0}, 00-01}^{R}$ has a maximum near $J=0$ for small $E$ with the peak gradually shifting to small positive $J$ as $E$ is increased. Furthermore, the number of values of $J$ which must be included, in order that the differential reaction cross sections [see Eq. (5.30) of Paper I] should have converged to within approximately $2 \%$, increases with $E$ from about 9 at $E=0.30 \mathrm{eV}$ (i.e., $|J| \leq 4$ ) to about 23 at $E=0.65 \mathrm{eV}$ $(|J| \leq 11)$. The maximum in the reaction probabilities at small $J$ indicates that only small impact parameter collisions contribute significantly to the reaction cross section. The semiclassical relation between the impact parameter $b$ and the orbital angular momentum $l$ [as given by Eq. (5.21) of I] is

$$
b=l / k_{v j} \text {, }
$$

where we define the sign of $b$ to be the same as that of $l$, and $k_{v g}$ is the wave number associated with the incident state. Since $l=J-j$, and $j=0$ for the transitions considered in Figs. 1 and 2, we see that $b$ is proportional to $J$, and thus the range of impact parameters which contribute significantly to the reaction cross section increases with $E$ in those figures (from $|b| \leq 1.74$ bohr at $0.45 \mathrm{eV}$ to $|b| \leq 2.22$ bohr at $0.65 \mathrm{eV}$ ). In Fig. 3 we plot the reaction probabilities vs $J$ at several energies for the transition $v=0, j=0 \rightarrow v^{\prime}=0, j^{\prime}=0$. Equation (3.1) indicates that this transition probability should be symmetric about $J=0$, but aside from that 


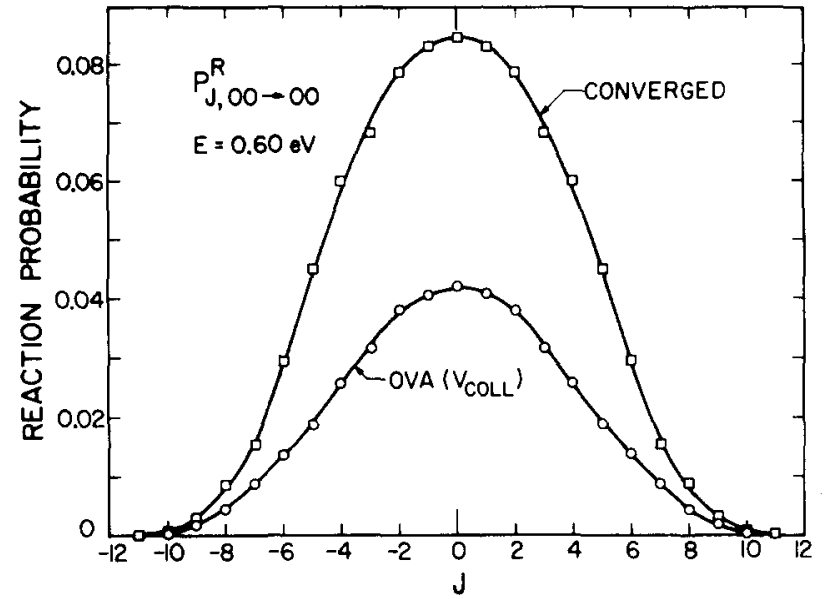

FIG. 4. Coplanar reaction probability $P_{J, 00-00}^{R}$ for $E=0.60 \mathrm{eV}$ (translational energy $E_{0}=0.33 \mathrm{eV}$ ) vs the total angular momentum quantum number $J$. Squares indicate the converged result while circles indicate the OVA probability for the collinear reference potential $\left[V_{\text {coll } 1}=V\left(r_{\lambda}, R_{\lambda}, \gamma_{\lambda}=0\right) \mid\right.$.

restriction, we find that the curves in that figure are otherwise very similar in appearance to those in Fig. 2. This conclusion applies quite generally to the reaction probability vs $J$ plots obtained for most other reactive transitions. A discussion of the energy dependence of the reaction probabilities will be given in Sec. III. D.

In Fig. 4 we compare the converged reaction probabilities $P_{J, 00-00}^{R}$ with the corresponding OVA results for a collinear reference potential $V_{\text {col1 }}=V^{\lambda}\left(r_{\lambda}, R_{\lambda}\right.$, $\gamma_{\lambda}=0$ ) at an energy of $0.60 \mathrm{eV}$. We see that the OVA result has the correct functional dependence on $J$ but that the magnitudes of the probabilities at each $J$ are nearly a factor of 2 too small. OVA calculations using $V_{\text {ref }}$

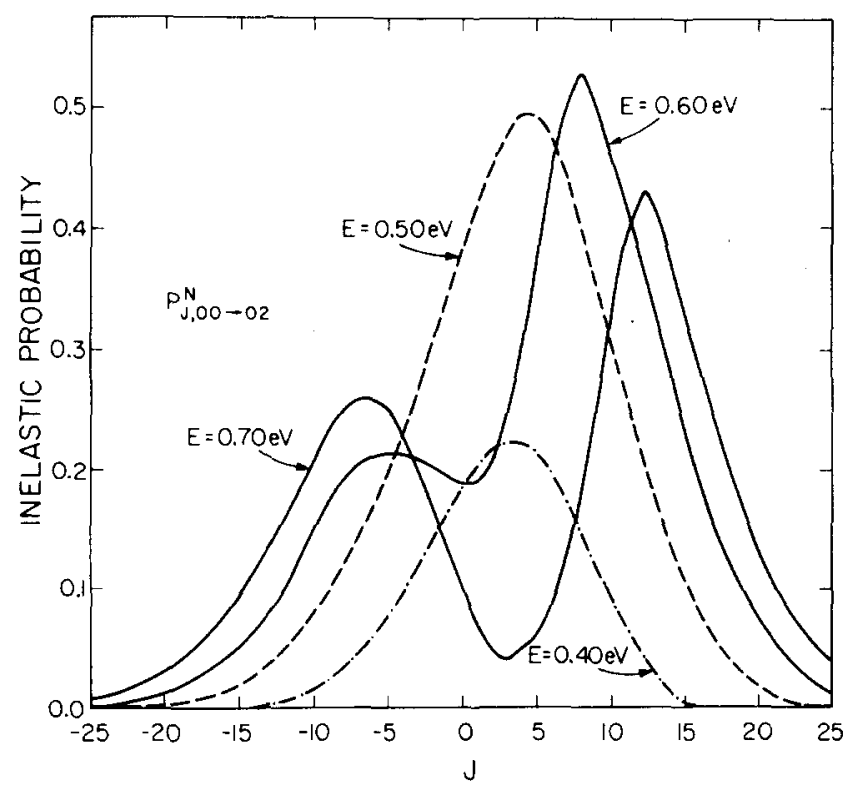

FIG. 5. Coplanar converged inelastic probability $P_{J, 00-02}^{N}$ as a function of $J$ for total energies $E=0.40 \mathrm{eV}$ (dash-dot), $0.50 \mathrm{eV}$ (dashed), $0.60 \mathrm{eV}$ (solid), and $0.70 \mathrm{eV}$ (solid).

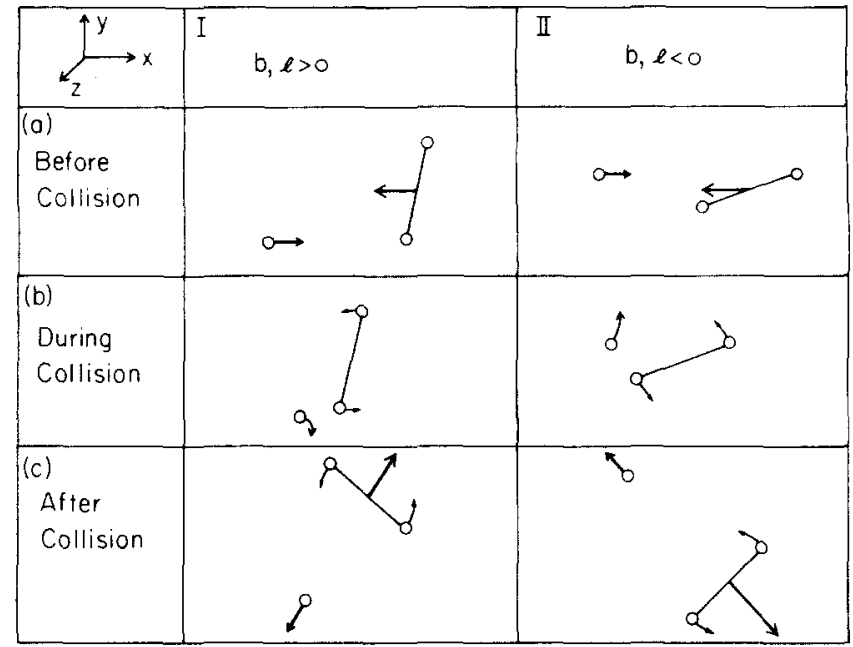

FIG. 6. Schematic representation of dominant collisions contributing to the $j=0 \rightarrow j^{\prime}>0$ collisional (nonreactive) excitation process. Shown are (a) the initial approach of the collision partners in the center of mass coordinate system, (b) the collision itself with the direction of the rotational polarization indicated by curved arrows, and (c) the receding scattered particles. Collision I considers $l$ (and hence $b$ ) initially positive (relative to the coordinate system shown). Collision II considers negative initial $l$ and $b$. Note that the diatomic rotor with $j=0$ is classically motionless with equal probability for any rotational phase. The particular phase chosen was that believed to give significant rotational excitation for each situation pictured.

$=V_{0}^{\lambda}\left(r_{\lambda}, R_{\lambda}\right)$ yield probabilities which are only slightly different from the OVA results in Fig. 4. (For example, the $V_{\text {ref }}=V_{0}^{\lambda}$ OVA reaction probability for $J=0$ is 0.0397 compared to 0.0420 in Fig。4). The analogous comparison at other energies between 0.3 and $0.6 \mathrm{eV}$ indicates that the OVA probabilities for the two choices of $V_{\text {ref }}$ always have values within $30 \%$ of one another. More important, the OVA probabilities and converged results are generally in good agreement in their $J$ dependence, but in very poor agreement in energy dependence, differing by several orders of magnitude at low energies. This difference in energy dependence has a dominant influence on the behavior of the reaction cross sections, as will be discussed in Sec. III. C.

In Fig. 5 we plot the inelastic nonreactive probabilities for the transition $v=0, j=0-v^{\prime}=0, j^{\prime}=2$ vs $J$ for several energies $E$. The inelastic probabilities are seen to span a much larger range of $J$ 's than the reactive ones, indicating that larger impact parameter collisions can contribute significantly to the inelastic processes. At all energies in Fig. 6, the maximum rotational excitation probability occurs for $J$ positive (although a smaller magnitude negative $J$ peak does appear at the higher energies). The increased likelihood of exciting a positive rotational sublevel in a nonreactive collision with $J$ initially positive is in agreement with the classical picture of the collision shown in Fig. 6 (Collision I) in which the incident atom having a positive impact parameter [see Eq. $(3,2)$ ] impulsively strikes the "bottom" atom of the diatomic molecule in Fig. 6, I(a), thus exerting positive torque on that 


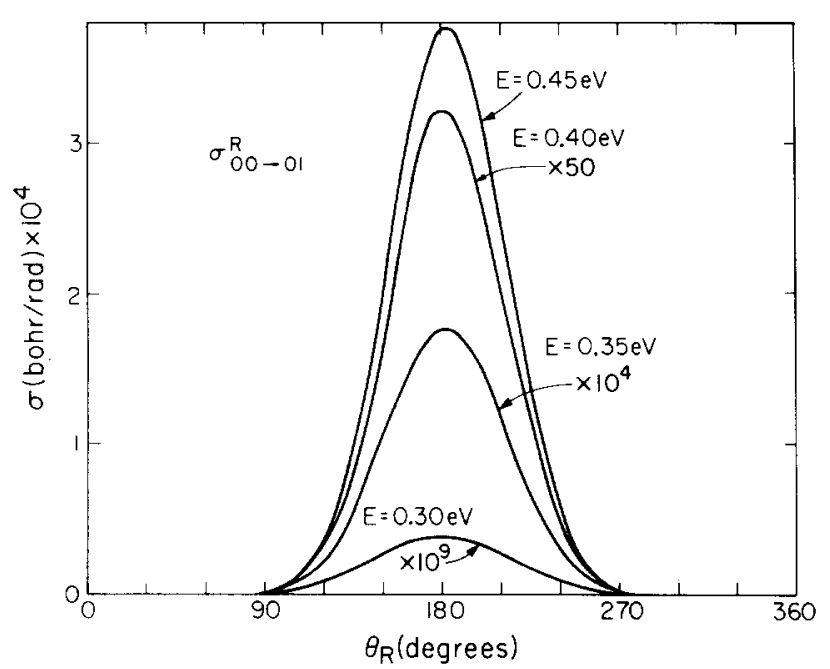

FIG. 7. Converged coplanar reactive differential cross section $\sigma_{00}^{R}-01$ vs the scattering angle $\theta_{R}$ for total energies $E=0.30$, $0.35,0.40$, and $0.45 \mathrm{eV}$.

molecule and exciting it into a rotational state with positive $j^{\prime}$. For this collision, one would expect the dominant scattering angle $\theta$ to lie between $180^{\circ}$ and $360^{\circ}$ relative to the $y$ axis of Fig. 6 . In the next section we shall see that this is precisely what the differential cross sections indicate. Still unexplained, however, are the double-peaked distributions at the higher energies in Fig. 5. Intuitively, one would expect that the positive $J$ peak results from the mechanism described above (Collision I in Fig. 6). The negative $J$ peak must arise from a different collision mechanism, quite possibly that pictured in Collision II of Fig. 6, in which the incident atom, having small negative impact parameter, still strikes the bottom atom of the diatom in Fig. 6 , IIa, but rebounds into the $0^{\circ} \leq \theta \leq 180^{\circ}$ hemisphere.

\section{B. Differential cross sections}

Figures 7,8 , and 9 depict the differential reactive cross sections corresponding to the same transitions and energy ranges as were used for the reaction prob-

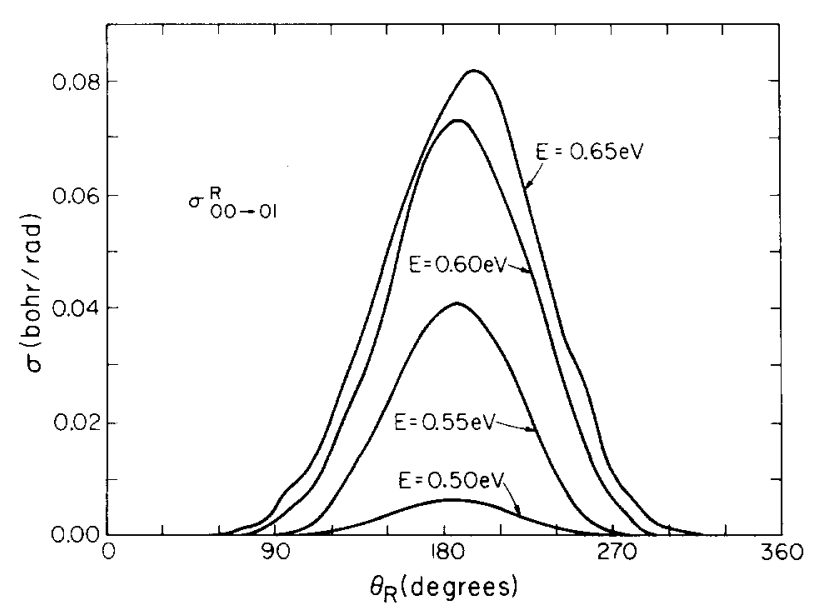

FTG. 8. Converged coplanar reactive differential cross section $\sigma_{00}^{R} \rightarrow 1$ as a function of the scattering angle $\theta_{R}$ analogous to Fig. 7 but at total energies $E=0.50,0.55,0.60$, and $0.65 \mathrm{eV}$.

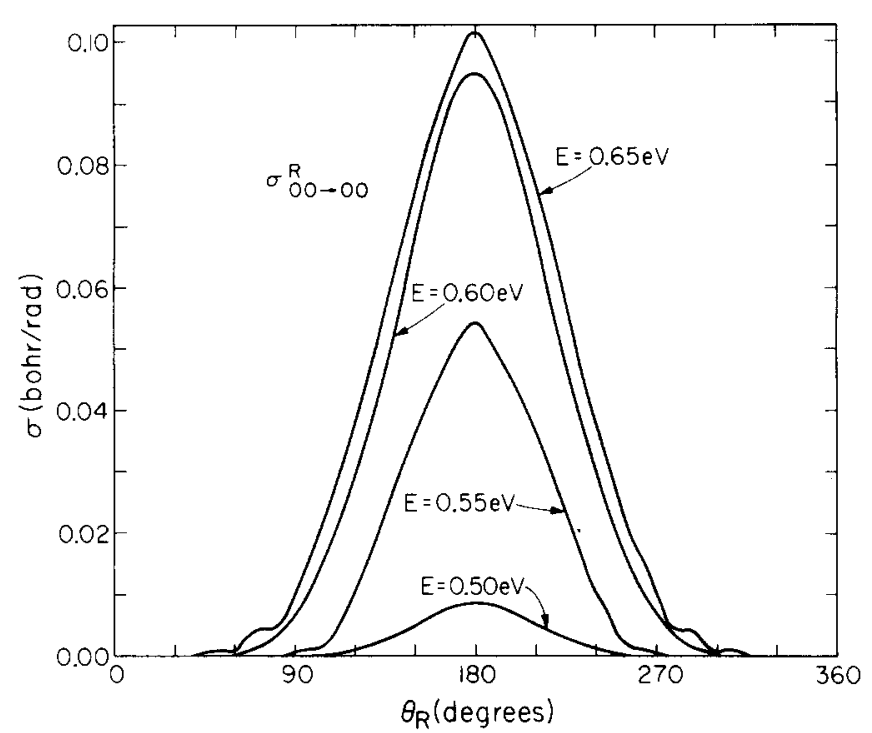

FIG. 9. Converged coplanar reactive differential cross section $0_{00}^{R} \rightarrow 00$ vs scattering angle $\theta_{R}$ at same energies as in Fig. 8. These curves are symmetric about $\theta_{R}=180^{\circ}$.

abilities in Figs. 1, 2, and 3, respectively. The $v=0$, $j=0 \rightarrow v^{\prime}=0, j^{\prime}=1$ distinguishable-atom reactive cross sections of Figs. 7 and 8 can be trivially converted to the corresponding antisymmetrized para - ortho quantities by multiplication by 3 [see (Eq. 2.1)]. Because of Eq. (2.3), the curves in Fig. 9 are exactly symmetric about $\theta_{R}=180^{\circ}$. We see that all reactive differential cross sections are strongly backward peaked. This is in agreement with the results of three- and two-dimensional quasiclassical calculations ${ }^{1,2}$ and with the results of experiments on $\mathrm{D}+\mathrm{H}_{2}{ }^{26}$ and $\mathrm{H}+\mathrm{T}_{2},{ }^{27}$ and is consistent with a rebound-type collision mechanism. The magnitudes of the differential cross sections near $\theta_{R}=0^{\circ}$ are all sufficiently small to allow us to conclude that forward scattering contributions to the reactive angular distributions are negligible. The small-amplitude oscillations in some of the higher energy differential cross sections in Figs. 8 and 9 are very likely spurious since they typically result from incomplete interference between different partial waves. This type of oscillatory behavior can be caused by as little as a $5 \%$ relative error in the matrix elements of $\mathbf{S}_{J}$ for a single partial wave, thus pointing out that equally accurate calculations for each partial wave (even those contributing relatively little to the integral cross sections) are necessary if spurious effects of this type are to be avoided. Of course, if there were rotational resonances in certain partial waves, then we would properly expect to see some form of oscillatory behavior in the angular distributions. Rotational resonances have indeed been observed in calculations on nonreactive atom diatom scattering, but these resonances have always been associated with attractive wells in the potential surfaces used. ${ }^{28}$ In the case of the purely repulsive Porter-Karplus ${ }^{19}$ potential, such wells do not exist, and thus purely rotational resonances are unlikely. ${ }^{29}$ The full-width at half-maximum (FWHM) of the backward-scattered peak in the differential cross section remains relatively constant over the energy range studied and roughly equal to $70^{\circ}$ (i.e., $145^{\circ} \leq \theta_{R} \leq 215^{\circ}$ ) 


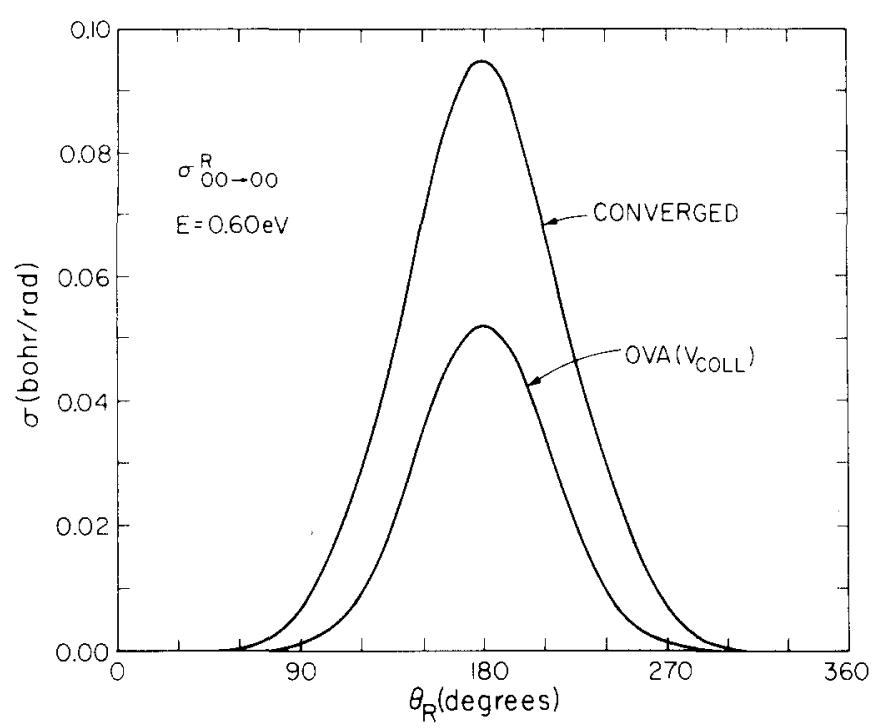

FIG. 10. Vibrationally converged and OVA differential cross section $\sigma_{00 \rightarrow 00}^{R}$ as a function of scattering angle $\theta_{R}$ at $E=0.60 \mathrm{eV}$ $\left(E_{0}=0.33 \mathrm{eV}\right)$. The OVA cross section was computed with a collinear reference potential $\left[V_{\text {col } 1}=V\left(r_{\lambda}, R_{\lambda}, \gamma_{\lambda}=0\right)\right]$. The cross section for this transition is symmetric about $\theta_{R}=180^{\circ}$.

in Fig. 9. Some broadening does, however, occur at the higher energies.

The angular distributions for the OVA results previously considered in Fig. 4 are plotted in Fig. 10. As in Fig. 4, we see that the shape of the converged curve is qualitatively well approximated by that of the OVA one, but there is about a factor of 2 difference in the magnitudes of the cross sections. This similarity in shape continues to exist at other energies as well, but the differences in magnitude can become much larger, as discussed in Sec. III. C.

In Fig. 11 we plot the distinguishable atom nonreactive inelastic differential cross sections $\sigma_{00 \rightarrow 02}^{N}$ at $E$ $=0.40,0.50,0.60$, and $0.70 \mathrm{eV}$. These angular dis-

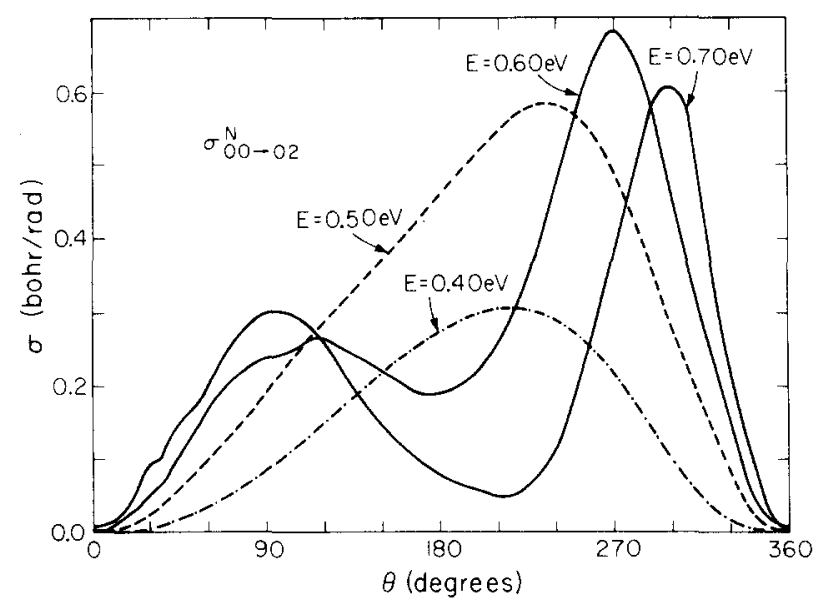

FIG. 11. Inelastic nonreactive (converged) differential cross section $\sigma_{00-02}^{N}$ for the coplanar $v=0, j=0 \rightarrow v^{\prime}=0, j^{\prime}=2$ transition as a function of scattering angle at total energies $E=0.40$ $\mathrm{eV}$ (dash-dot), $0.50 \mathrm{eV}$ (dashed), $0.60 \mathrm{eV}$ (solid), and $0.70 \mathrm{eV}$ (solid).

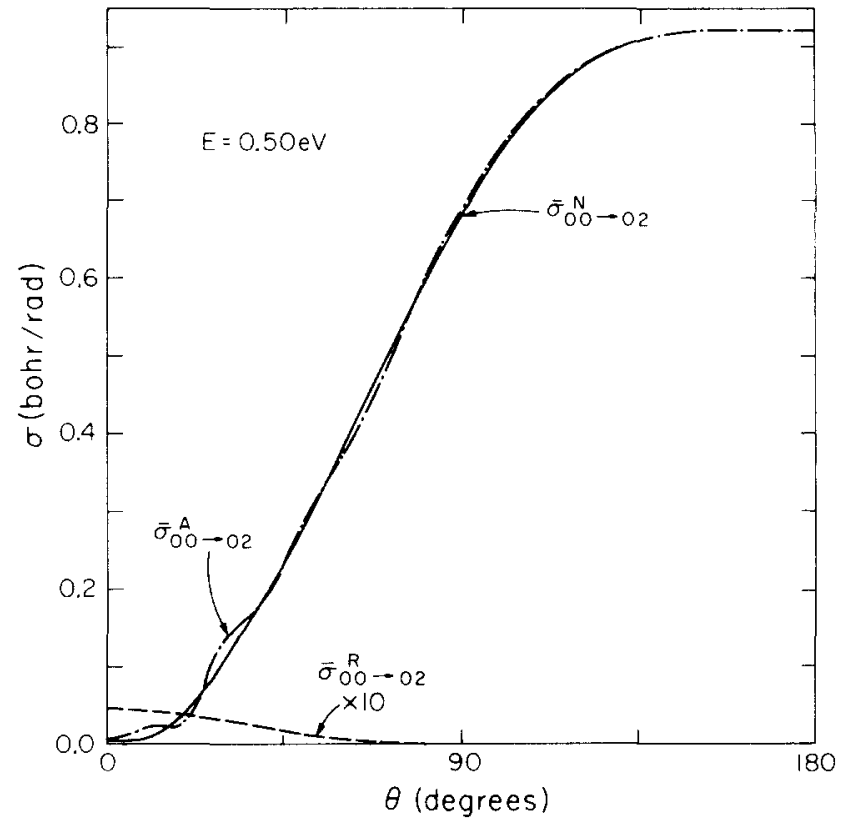

FIG. 12. Nonreactive (solid), reactive (dashed), and antisymmetrized (dash-dot) differential cross sections as a function of scattering angle for the coplanar $v=0, j=0 \rightarrow v^{\prime}=0,\left|j^{\prime}\right|=2$ transition (summed over final rotational polarizations) at $E$ $=0.50 \mathrm{eV}\left(E_{0}=0.23 \mathrm{eV}\right)$. Note that the scattering angle used is $\theta$ and $\theta=0$ corresponds, for reactive scattering, to $\theta_{R}=180^{\circ}$ [see Eq. $(2.5)$ ].

tributions clearly reflect the one- or two-peaked nature of the nonreactive probability plots of Fig. 5 and show predominantly backward to sidewards peaking with the position of the maximum shifting gradually to a more forward direction with increasing energy. The maximum value of $\sigma_{00.02}^{N}$ always occurs for $180^{\circ} \leq \theta \leq 360^{\circ}$, in agreement with our qualitative classical ideas of Sec. III. A. At higher energies we see double-peaked distributions, possibly corresponding to the two mechanisms pictured in Fig. 6. There is little evidence of any high frequency oscillations in any of the cross sections plotted in Fig。11, which indicates that the collision process is predominantly direct (nonresonant). The small-amplitude oscillations occurring at $\theta<100^{\circ}$ for $E=0.60$ and $0.70 \mathrm{eV}$ are probably spurious and of same origin as those for the reactive cross sections in Figs. 8 and 9.

Since the $v=0, j=0 \rightarrow v^{\prime}=0, j^{\prime}=2$ transition considered in Fig. 11 corresponds to a para-to-para transition which can occur by both nonreactive and reactive mechanisms, the correct physically measurable quantity (in a 2D world) to consider is the antisymmetrized parato-para cross sections which can be obtained through the use of Eq. (2.1). In Figs. 12, 13, and 14 we plot the resulting antisymmetrized angular distributions $\bar{\sigma}_{00 \rightarrow 02}^{A}$ (summed over degenerate product rotational polarizations) for total energies of $0.5,0.6$, and $0.7 \mathrm{eV}$, respectively. Also plotted for comparison are the corresponding distinguishable-atom nonreactive and reactive cross sections where, for consistency, the angle $\theta$ rather than $\theta_{R}$ [see Eq. (2.5)] is used for plotting the reactive differential cross sections. In terms of $\theta$, the reactive cross section is forward peaked (i.e., back- 


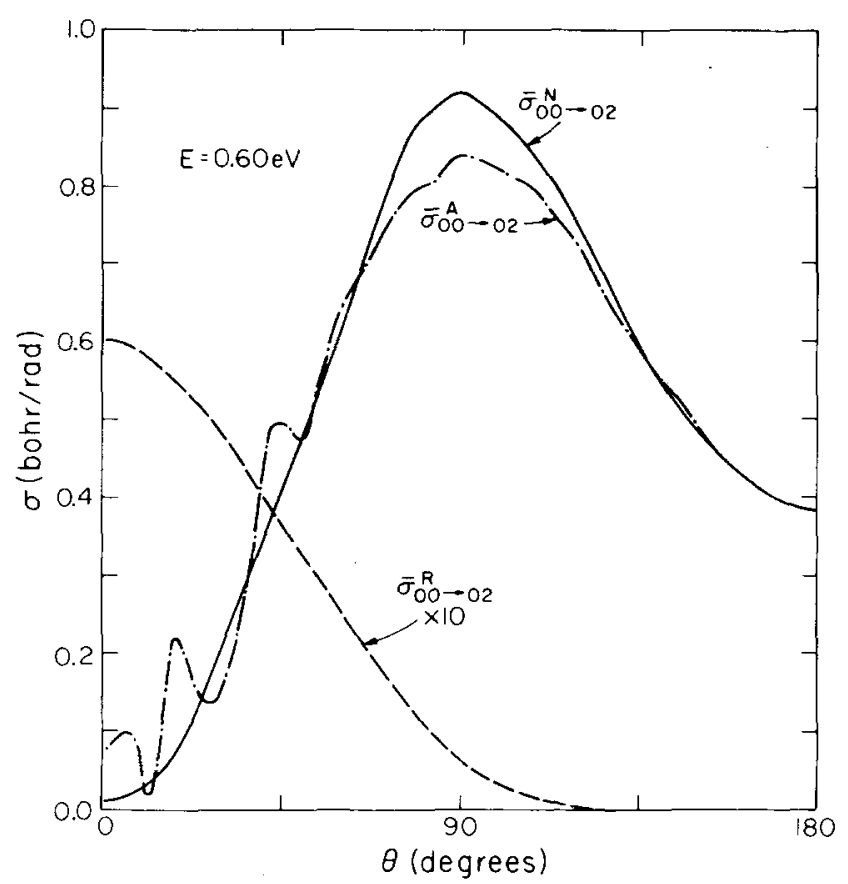

FIG. 13. Nonreactive, reactive, and antisymmetrized differential cross sections analogous to Fig. 12 at $E=0.60 \mathrm{eV}\left(E_{0}\right.$ $=0.33 \mathrm{eV})$. See remark about $\theta$ in the caption for that figure.

ward peaked in terms of $\theta_{R}$ ), while the nonreactive one is backward peaked at $0.5 \mathrm{eV}$ shifting to sidewards peaking at the higher energies. At $0.50 \mathrm{eV}$ (Fig. 12), the reactive cross section has a maximum value of $0.0045 \mathrm{bohr} / \mathrm{rad}$, which is over 200 times smaller than the maximum value of the nonreactive one, $0.92 \mathrm{bohr} /$ rad. This implies that $f_{v y-v^{\prime} f^{\prime}}^{N}$ in Eq. (2.1) has a much larger absolute value than $f_{v f \rightarrow v^{\prime} j^{\prime}}^{R}$, so that the antisymmetrized and nonreactive differential cross sections are

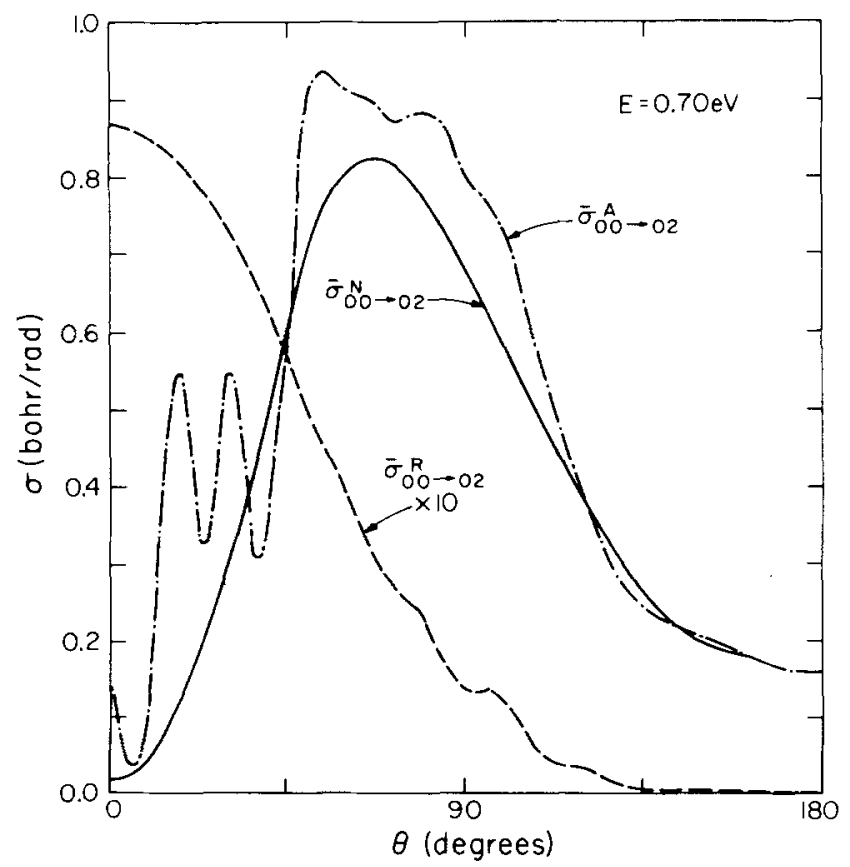

FIG. 14. Nonreactive, reactive, and antisymmetrized differential cross sections analogous to Fig. 12 at $E=0.70 \mathrm{eV}\left(E_{0}\right.$ $=0.43 \mathrm{eV})$. See remark about $\theta$ in the caption of that figure.

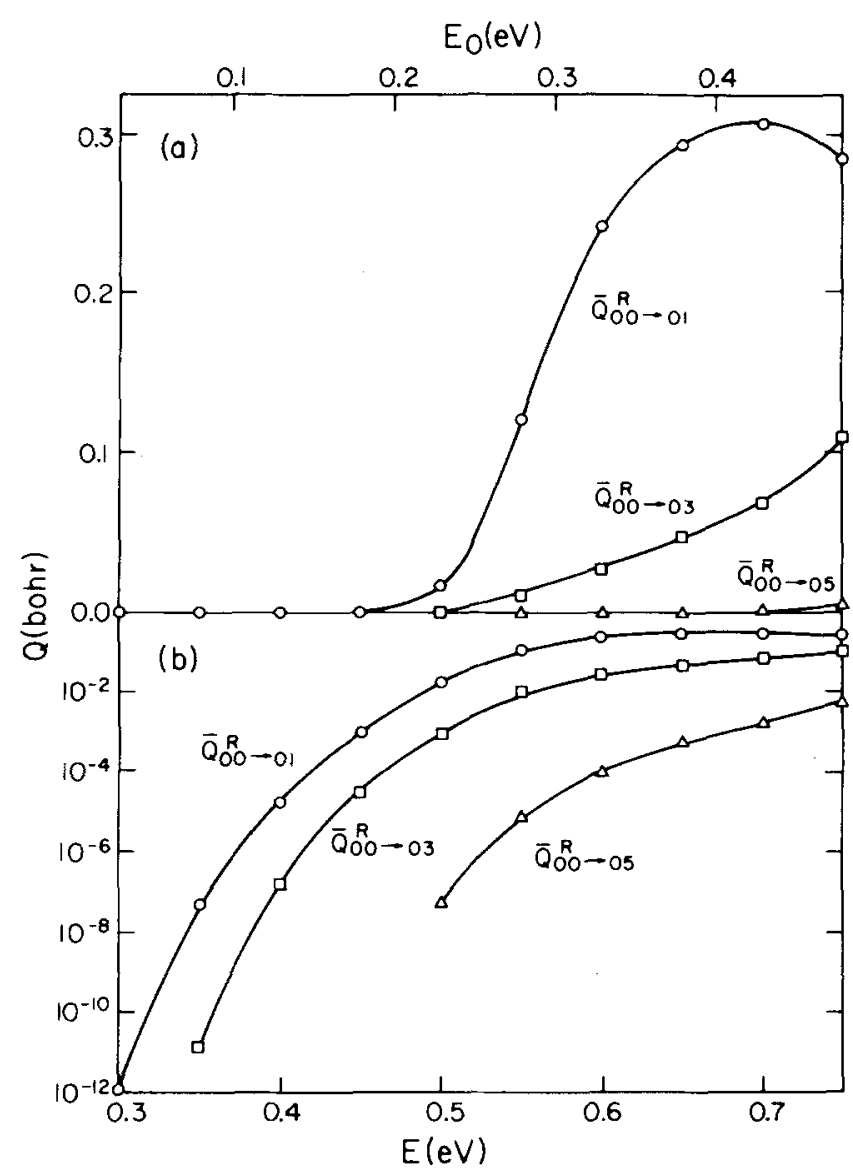

FIG. 15. Converged integral reactive cross sections $\bar{Q}_{00-01}^{R}$, $\bar{Q}_{00}^{R} \rightarrow 03$, and $\bar{Q}_{00-05}^{R}$ (summed over final rotational polarizations) vs total energy $E$ and translational energy $E_{0}$ : (a) linear scale, (b) semilogarithmic scale.

nearly identical. Some small amplitude oscillations are seen in $\bar{\sigma}_{00 \rightarrow 02}^{A}$ at small $\theta$ in the neighborhood of the region where the reactive cross section has a maximum. These oscillations are real and result from interference between the direct and exchange contributions to the antisymmetrized cross section. They are similar in origin to the quantum symmetry oscillations which have been observed in atom-atom elastic and inelastic scattering. ${ }^{30}$ As the energy is increased, the reactive cross sections increase much more rapidly than do the nonreactive ones (at all scattering angles), and (as is indicated in Figs. 13 and 14) the oscillations in the antisymmetrized differential cross sections for $\theta<60^{\circ}$ become quite pronounced in the forward $\theta$ (backward $\theta_{R}$ ) direction. (The oscillations in the antisymmetrized curve of Fig. 14 at $\theta>80^{\circ}$ correlate with those in the reactive curve. As for Figs. 8 and 9, the latter are probably spurious, and therefore the former should not be considered to be real either.) A small increase in the oscillation frequency with increasing energy is also apparent from the figures.

\section{Integral cross sections}

In Fig. 15 we plot the reactive integral cross sections $\bar{Q}_{00 \rightarrow 01}^{R}, \bar{Q}_{00_{-03}}^{R}$, and $\bar{Q}_{00+05}^{R}$ (summed over final rotational polarizations) as a function of the total energy $E$ and initial translational energy $E_{0}$. Both linear and 


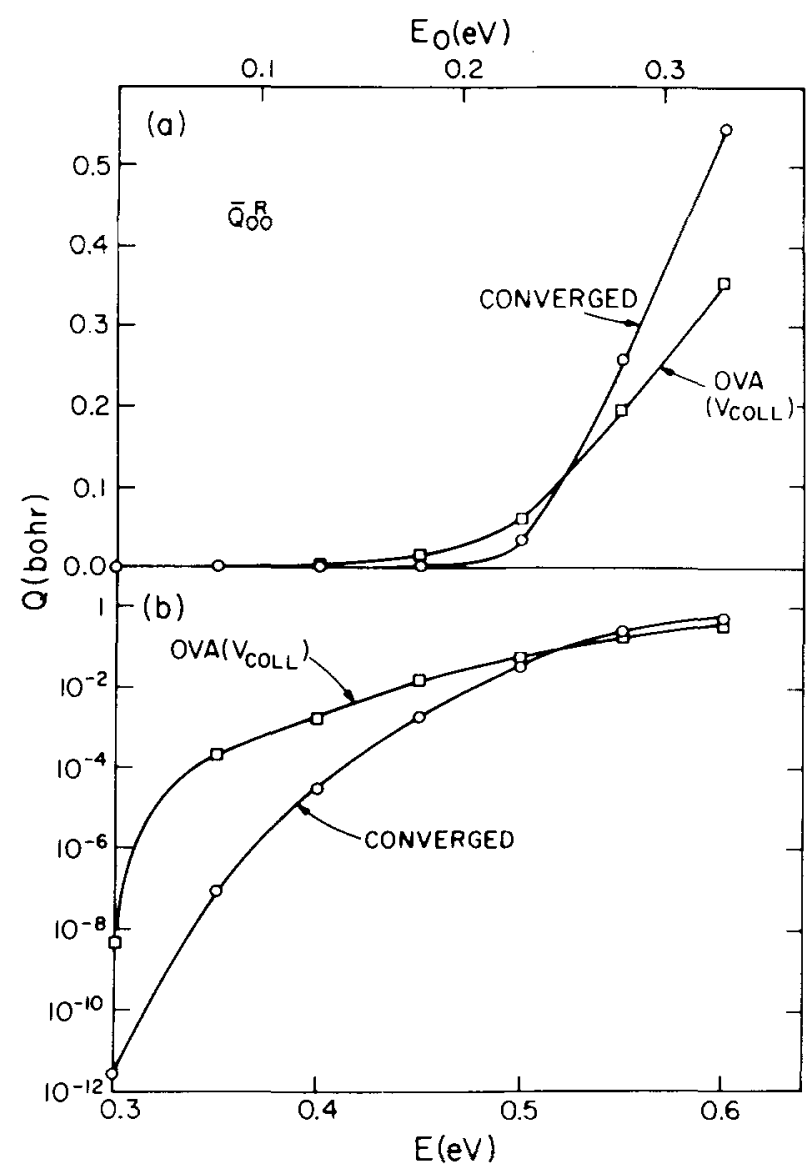

FIG. 16. Integral reactive cross section $\bar{Q}_{0}^{R}$ (summed over all accessible final states) vs total energy $E$ and relative energy $E_{0}$. Circles represent vibrationally converged results and squares the OVA ones using a collinear reference potential: (a) linear scale, (b) semilogarithmic scale.

semilogarithmic scales are used to show the functional dependence of these cross sections over a wide range of energies. If we define the effective threshold energy for a process as being that value of $E$ for which the corresponding integral cross section is $0.01 \mathrm{bohr}$, then the threshold energies for $\bar{Q}_{00 \uparrow 01}^{R}, \bar{Q}_{00 \uparrow 03}^{R}$, and $\bar{Q}_{00 \rightarrow 05}^{R}$ are $0.49 \mathrm{eV}, 0.55 \mathrm{eV}$, and $>0.75 \mathrm{eV}$, respectively. A discussion of the significance of the effective threshold energies will be deferred to $\mathrm{Sec}_{\mathrm{a}}$ III。 $\mathrm{D}$, where we also compare the coplanar results with those of collinear calculations on the same potential energy surface. Above threshold, $\bar{Q}_{00-01}^{R}$ rises in a nearly linear manner with $E$ up to about $0,65 \mathrm{eV}$ and achieves a maximum value of 0.31 bohr at about $E=0.70 \mathrm{eV} . \bar{Q}_{00 \rightarrow 03}^{R}$ and $\bar{Q}_{00 \rightarrow 05}^{R}$ increase monotonically in the energy range spanned by this figure but may level off at higher energies. At very low energies, the integral cross sections exhibit approximate exponential dependence on $E$. A characterization of the product rotational state distribution implicit in Fig. 15 is given in Sec. III. E. We should finally note that the reactive cross sections in Fig. 15 can be converted to the corresponding para $\rightarrow$ ortho quantities by multiplication by 3 .

In Fig. 16 we compare the reactive cross section $\bar{Q}_{00}^{R}$ (summed over all product states) with the OVA re- sult [using $V_{\text {ref }}=V\left(r_{\lambda}, R_{\lambda}, \gamma_{\lambda}=0\right)$ ]. As mentioned in Sec. III. A, the results obtained using $V_{\text {ref }}=V_{0}^{\lambda}\left(r_{\lambda}, R_{\lambda}\right)$ have almost the same energy dependence. It is apparent from the figure that the vibrationally converged integral cross section differs quite significantly from the OVA result over much of the energy range considered, the difference being about 3 orders of magnitude for total energies below $0.36 \mathrm{eV}$. The two curves do cross near $E=0,52 \mathrm{eV}$, which is quite interesting, since a previous analysis of the collinear $\mathrm{H}+\mathrm{H}_{2}$ system (on a slightly different potential surface) indicated that this reaction is very nearly vibrationally adiabatic at this energy. ${ }^{31}$ Since, as we shall see in the next section, collinear and coplanar calculations can be related in a reasonably accurate manner, one might be able to assess the accuracy of OVA calculations in two and three dimensions by analyzing the extent of vibrational adiabaticity in the corresponding collinear systems.

In Fig. 17 we compare the nonreactive and antisymmetrized integral cross sections $\bar{Q}_{00 \ldots 02}^{N}$ and $\bar{Q}_{00.02}^{A}$ as a function of $E$ and $E_{0}$. The rotationally inelastic cross sections have much larger magnitudes than the reactive ones of Figs. 15 and 16 with a peak value of 3.76 bohr near $E=0.54 \mathrm{eV}$. Since the $v^{2}=0, j^{\prime}=2$ state of $\mathrm{H}_{2}$ becomes energetically accessible at $E=0.30 \mathrm{eV}$, we see that there is essentially zero threshold energy for the nonreactive process so that $\bar{Q}_{00-02}^{A}$ coincides almost exactly with its distinguishable-atom counterpart $\bar{Q}_{00 \rightarrow 02}^{N}$ at all energies below $0.50 \mathrm{eV}$. Thereafter, $\bar{Q}_{00 \rightarrow 02}^{A}$ becomes progressively larger than $\bar{Q}_{00-02}^{N}$ with no apparent oscillatory behavior as a function of energy resulting, in contrast to the angular distributions of Figs. 12-14.

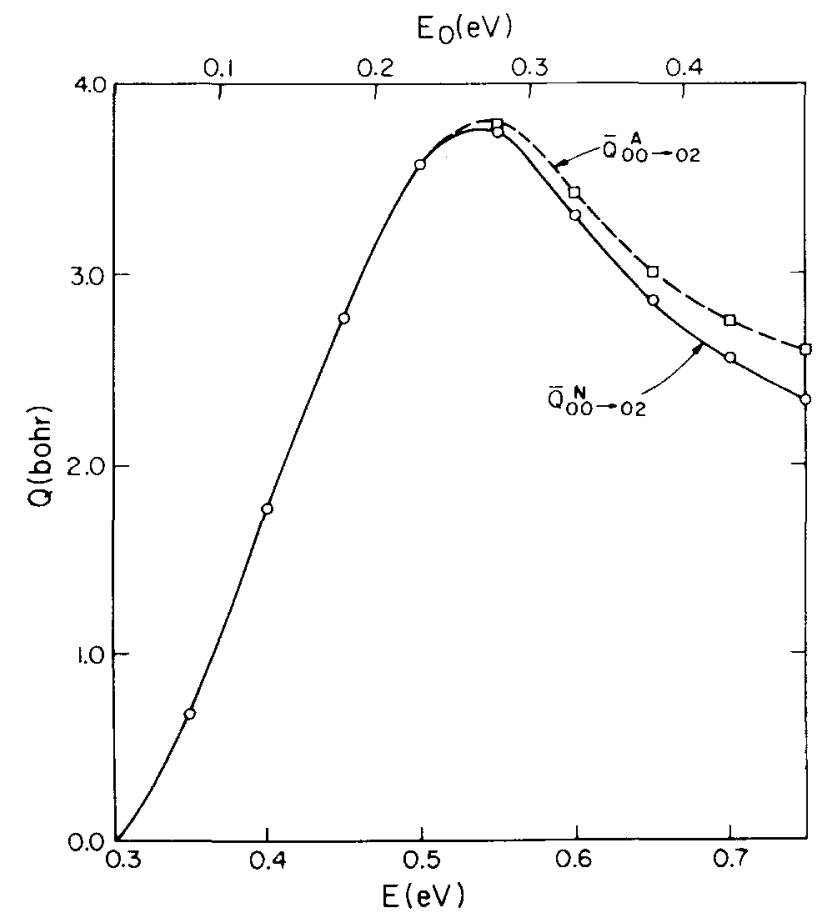

FIG. 17. Inelastic nonreactive integral cross sections $\bar{Q}_{00}^{N} \rightarrow 02$ and $\bar{Q}_{00 \rightarrow 02}^{A}$ (summed over final polarizations) vs the total energy $E$ and relative translational energy $E_{0}$. 


\section{Comparison of planar and collinear results}

Because planar cross sections have the dimensions of length while collinear ones are dimensionless (i.e., the collinear cross section is equal to the collinear reaction probability), a direct comparison of these quantities is not possible. One could devise models for converting collinear results into planar ones by assigning a model impact parameter dependence to the collinear reaction probabilities. A more straightforward comparison can be effected instead by examining the behavior of the corresponding collinear ${ }^{32,33}$ and planar reaction probabilities (the latter for $J=0$ ) as are plotted in Fig. 18. Probabilities for other $J$ 's or different initial $v j$ states could have been used, but those for $J=0$ and $v=j=0$ were chosen for this comparison because they correspond more closely to the collinear conditions. This choice is furthermore justified by the fact that the form of the energy dependence of the planar probabilities for different $J$ or $j$ (for reasonably small values of these quantum numbers) is essentially the same as that of $P_{00}^{R}(J=0)$, as is demonstrated in Fig. 19 [where $P_{00}^{R}(J=0), P_{00}^{R}(J=4)$, and $P_{01}^{R}(J=0)$ are plotted]。The $P_{00}^{R}(J)$ curves, for $J=1,2,3$, would all lie between the corresponding curves for $J=0$ and $J=4$. Figure 18 indicates that the collinear and coplanar results have nearly the same energy dependence, the en-

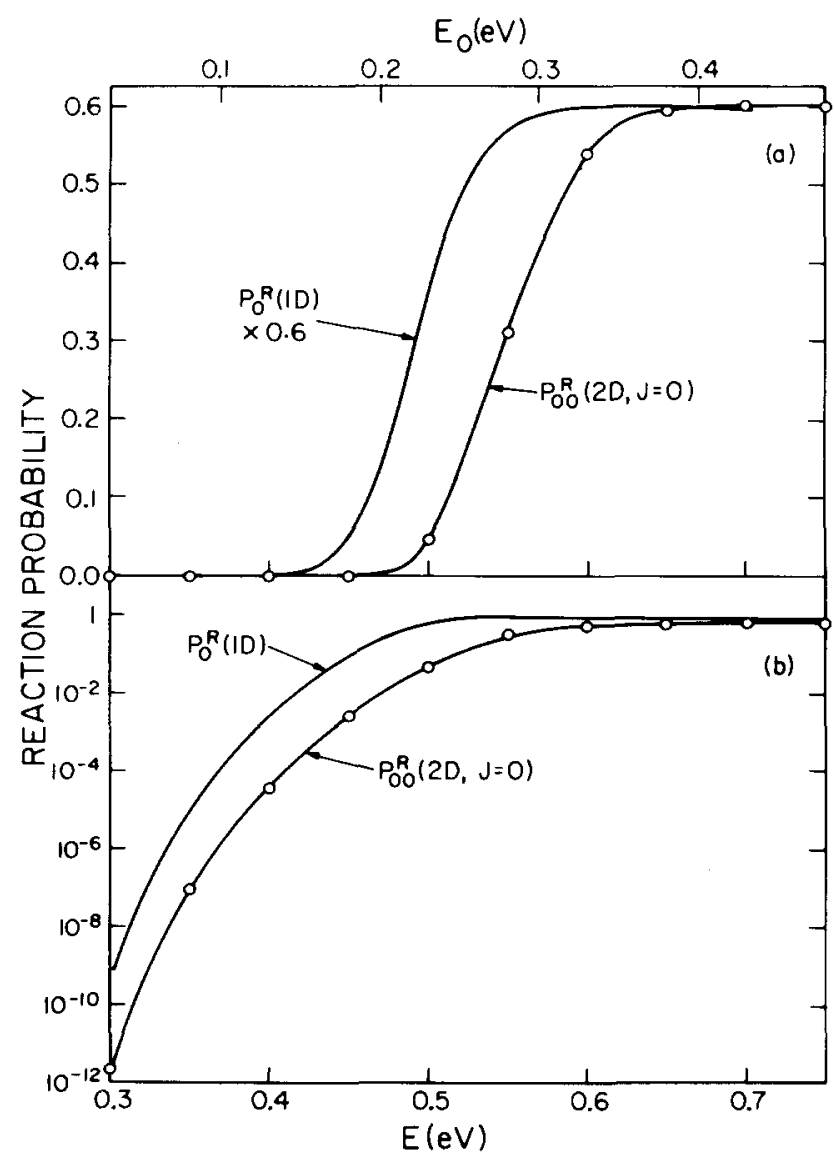

FIG. 18. Total reaction probabilities $P_{0}^{R}(1 D)$ (collinear) and $P_{00}^{R}(2 \mathrm{D}, J=0)$ (coplanar, $J=0, v=0, j=0$ and summed over all final states) vs the total energy $E$ and translational energy $E_{0}$ : (a) linear scale, with the collinear results multiplied by 0.6 ; (b) semilogarithmic scale.

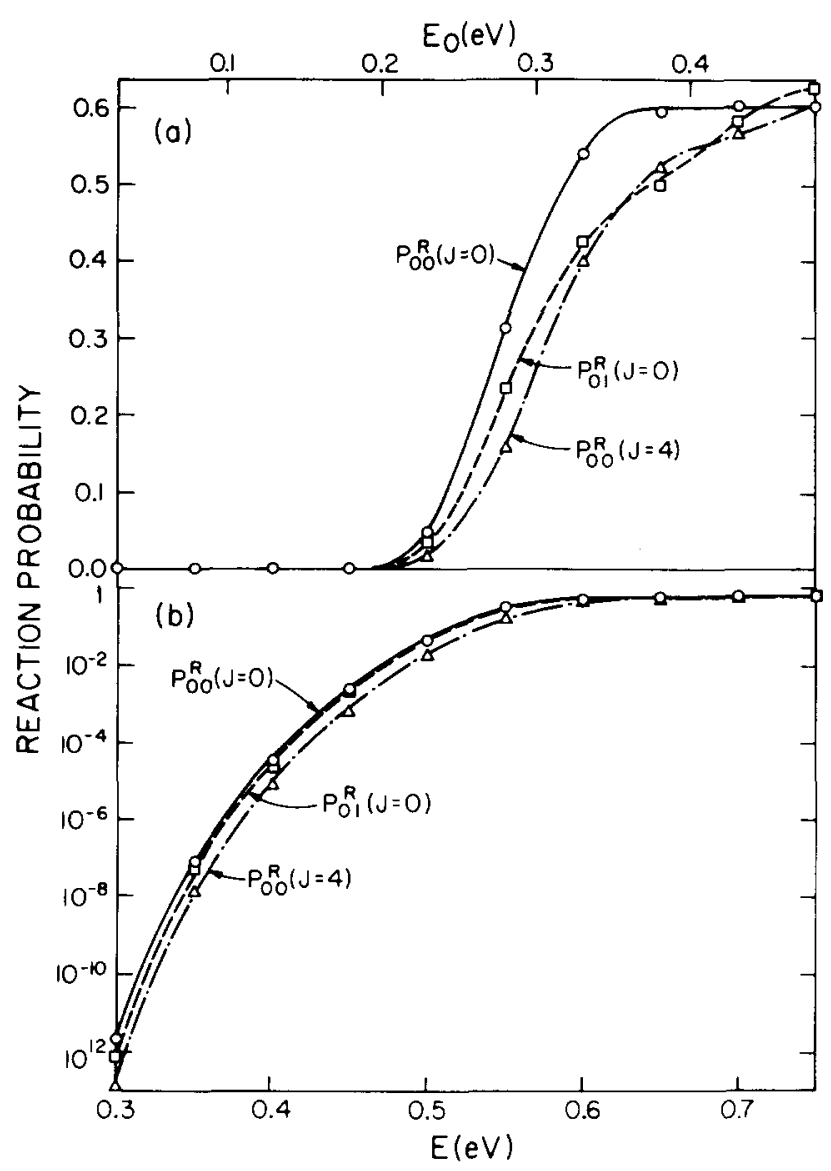

FIG. 19. Coplanar total reaction probabilities $P_{00}^{K}(J=0)$ (circles, solid curve), $P_{00}^{R}(J=4)$ (triangles, dash-dotted curve), and $P_{01}^{R}(J=0)$ (squares, dotted curve) summed over all final states vs total energy $E$ and translational energy $E_{0}$ : (a) linear scale, (b) logarithmic scale.

ergy scale being shifted upwards by about $0.055 \mathrm{eV}$ in going from the collinear to the coplanar curves. In addition, the maximum value of the collinear reaction probability is 1.0 , whereas that of the coplanar one is about 0.6 . Both the energy shift and the difference in the maximum probability are explainable in terms of relatively simple concepts. To understand the energy shift, we examine the nature of the triatomic $\mathrm{H}_{3}$ system in its transition state. In the linear case, this triatomic pseudomolecule has two vibrational degrees of freedom: an asymmetric stretch mode, which is unstable and leads to motion along the reaction coordinate, and a stable symmetric stretch mode. When the reaction occurs and the system passes through the transition state region, the total energy partitions itself between these two vibrational modes. Energy in the symmetric stretch mode is not easily converted into the asymmetric stretch mode making it unavailable to overcome the potential surface barrier. This is a partial physical interpretation of the fact that the collinear threshold energy (the value of $E$ at which the reaction probability is 0.01 ) is $0.42 \mathrm{eV}$, which is somewhat larger than the $0.396 \mathrm{eV}$ Porter-Karplus surface barrier height. Collinear threshold phenomena such as this have been analyzed in detail elsewhere. ${ }^{6,31}$ In going from a linear to a planar transition state we add one bending degree of freedom to the internal motion of the 


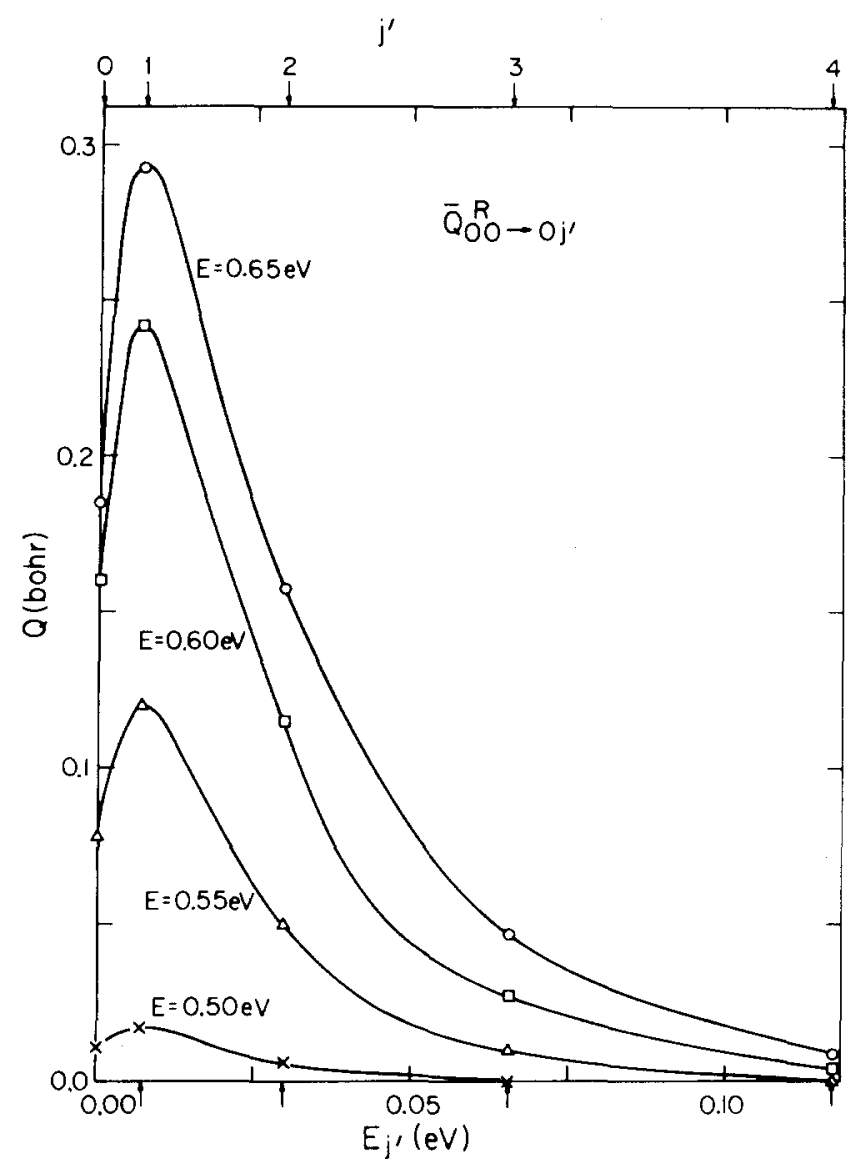

FIG. 20. Converged coplanar integral reaction cross sections $\bar{Q}_{00 \rightarrow 0 j}^{R}$, vs the final rotational energy at total energies $E=0.50$ $\mathrm{eV}$ (triangles), $0.55 \mathrm{eV}$ (triangles), $0.60 \mathrm{eV}$ (squares), and $0.65 \mathrm{eV}$ (circles). The arrows in lower and upper abcissa indicate the $E_{j}$, rotational energies. The curves are drawn smoothly through the points.

transition state which also does not contribuie effectively to motion along the reaction coordinate and which will also tie up some of the energy needed to overcome the activation barrier. This additional energy in the bending motion is a plausible explanation for the $0.055 \mathrm{eV}$ upward energy shift observed in Fig. 18 and is approximately equal to the zero point bending energy of about $0.06 \mathrm{eV}$ for the surface used. ${ }^{34}$ Much of the above explanation has its basis on an approximate statistical theory proposed by Marcus. ${ }^{35}$ The difference in the maximum probabilities attained by the collinear and coplanar results can be understood by examining the orientation dependence of the reaction probability. In the planar case with $j=0$ initially, the diatomic molecule does not rotate and has equal probability of being in any orientation with respect to the direction of approach of the incident atom. Since the barrier height of the potential energy surface is $0.396 \mathrm{eV}$ for collinear collisions and increases to $2.8 \mathrm{eV}$ for perpendicular ones, we would expect that in the energy range being considered, the reaction probability should be greater for linear collisions and decrease to zero for perpendicular ones. The coplanar probability should represent an average over all initial orientations and if we assume unit reaction probability for $0 \leq \gamma_{\lambda} \leq 54^{\circ}$ and $306^{\circ} \leq \gamma_{\lambda}$ $\leq 360^{\circ}$ and, by symmetry, for $\left|180^{\circ}-\gamma_{\lambda}\right| \leq 54^{\circ}$ and zero probability elsewhere, we obtain a coplanar reaction probability of 0.60 in agreement with Fig. 18. The $54^{\circ}$ cut-off angle is in reasonable agreement with previous estimates of the orientation dependence of the reaction probability obtained from distorted wave results ${ }^{15}$ and from classical trajectory results. ${ }^{1}$

\section{E. Product state rotational distributions}

In Fig, 20 we plot the integral cross sections $\bar{Q}_{00-0 j^{\circ}}$, (summed over final rotational polarizations) as a function of the production rotational energy and quantum number for several total energies $E$. We see from the figure that only small $j^{\prime}$ rotational states are appreciably excited in these reactive collisions. The relative population of final rotational states is not strongly dependent on total energy although some broadening of the distribution does occur at higher $E$. Not shown in the figure are the final rotational state distributions from initial states $j \neq 0$. The qualitative shapes of these distributions are not strongly dependent on $j$ and look very much like those for $j=0$ in Fig。 20. However, the magnitudes of the $\bar{Q}_{0_{j \rightarrow 0 j}}^{R}$, decrease monotonically with increasing $j$ for a given $j^{\prime}$. $^{36}$

To a large extent, the distributions in Fig. 20 resemble rotational Boltzman-like distributions with a single temperature parameter. Distributions of this type, for a planar system, may be expected to have the form $^{37}$

$$
f_{j^{\prime}}(E)=A(E)\left(2-\delta_{j^{\prime} 0}\right) e^{-E_{f^{\prime}} / k T(E)},
$$

where $A(E)$ and $T(E)$ are energy dependent constants and $2-\delta_{, 10}$ is a degeneracy factor. In Fig. 21 we plot $\left[2 /\left(2-\delta_{f, 0}\right)\right] \bar{Q}_{00 \rightarrow 0 f}^{R}$, as a function of the product rotational energy on a logarithmic ordinate scale. The resulting curves for different $E$ are approximately linear (most nearly so at the higher energies) in agreement with the predictions of Eq. (3.3), with temperature parameters $T(E)$ in the neighborhood of $250-400 \mathrm{~K}$. We should point out that although the rotational distributions are temperaturelike, we find no evidence of long lived compound state (i. e., complex) formation in this reaction at the energies being considered. ${ }^{32}$ The rotational distributions seem to be determined to a large extent by the shape of the potential energy surface in the transition state region of configuration space [see lower half of Fig. 2(c) of Paper I]. In this transition state, the asymptotic free rotational motion has become a seriously restricted bending motion. This bending motion becomes again a free rotational motion after the reaction, and, at least qualitatively, the distribution of different product rotational states appears to be determined by the overlap of this bending wavefunction and the asymptotic free rotor wavefunction. If this reasoning is correct, the resemblance of the rotational distribution in Figs. 20 and 21 to thermal distributions is at least partially coincidental. This phenomenon should, however, be quite common since restricted bending motion in the transition state region is a common feature of the potential energy surfaces for many reactions.

In Fig. 22 we plot the OVA cross sections in a man- 


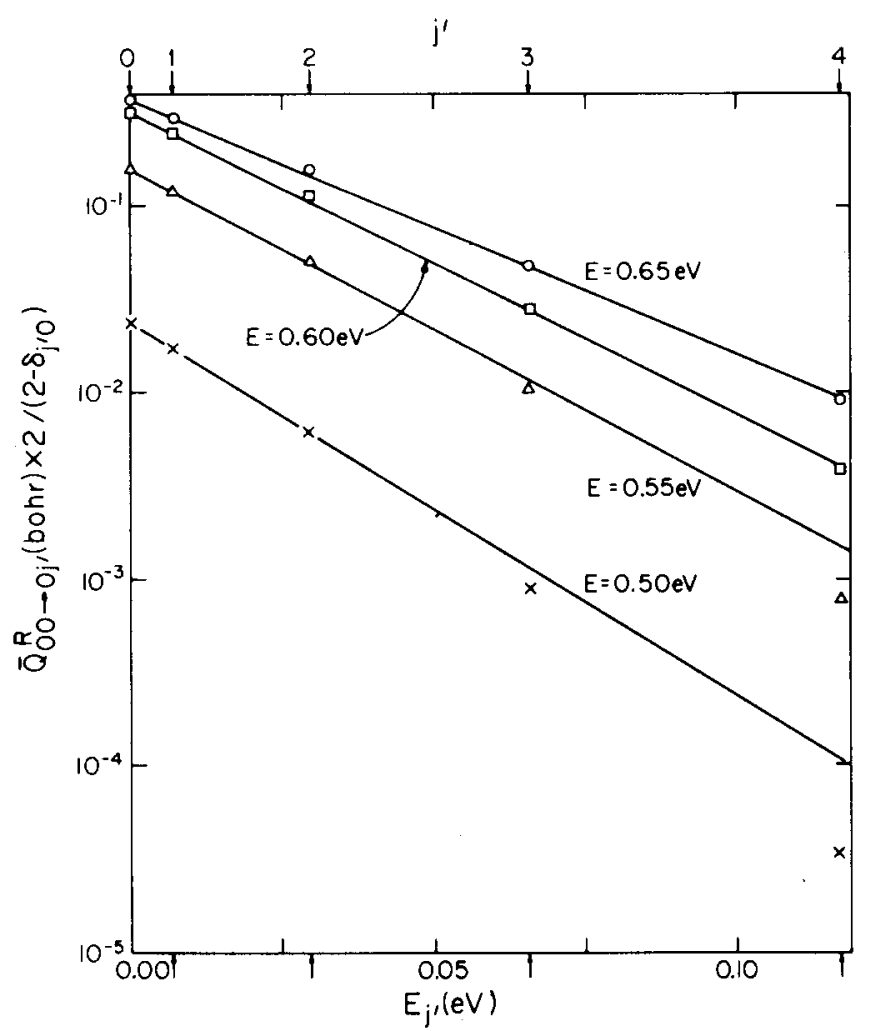

FIG. 21. Semilogarithmic plot of the integral reactive cross section $\bar{Q}_{00-0 j !}^{R}\left[\times 2 /\left(2-\delta_{j, 0}\right)\right]$ vs the final rotational energy at total energies $E=0.50,0.55,0.60$, and $0.65 \mathrm{eV}$ (symbols analogous to those of $\mathrm{Fig}$. 20). Straight lines are drawn connecting the low $j^{\prime}$ points. The arrows are as in Fig. 20 .

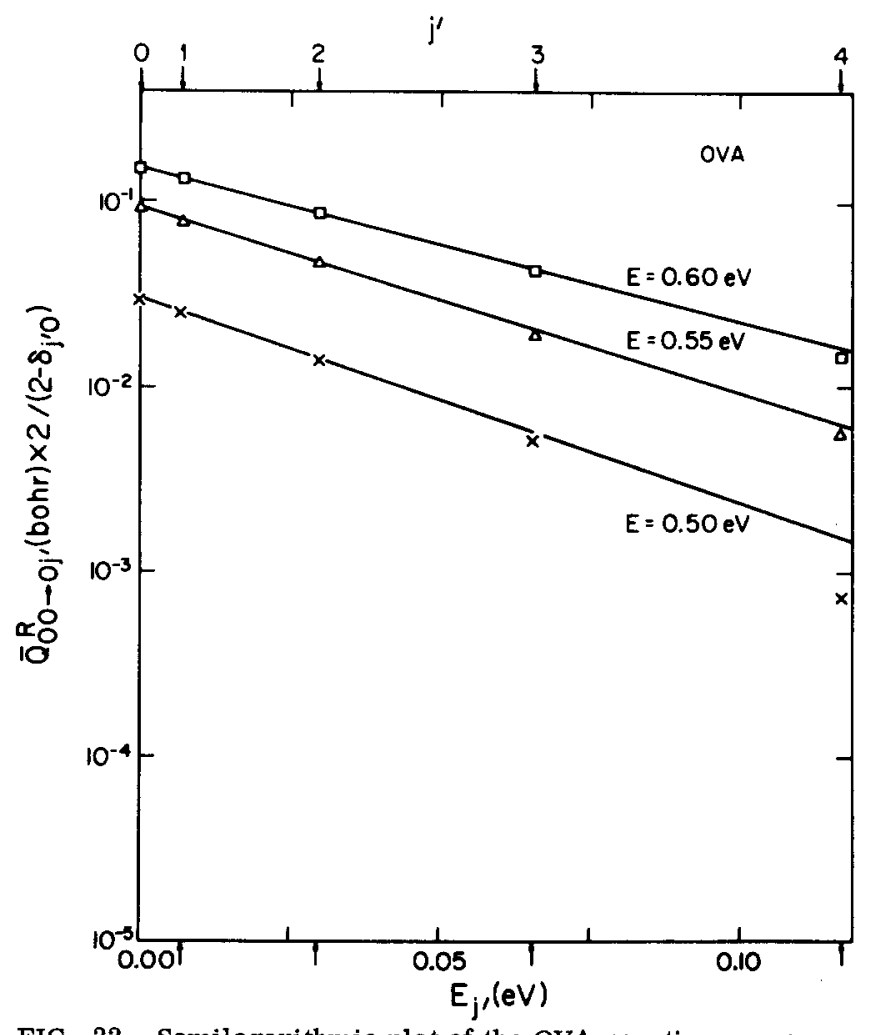

FIG. 22. Semilogarithmic plot of the OVA reactive cross section $\bar{Q}_{00-0 j}^{R},\left[\times 2 /\left(2-\delta_{j},_{0}\right)\right]$ as a function of the final rotational energy at energies $E=0.50,0.55$, and $0.60 \mathrm{eV}$. The straight lines drawn connect the low $j^{\prime}$ points. The arrows are as in Fig. 20.

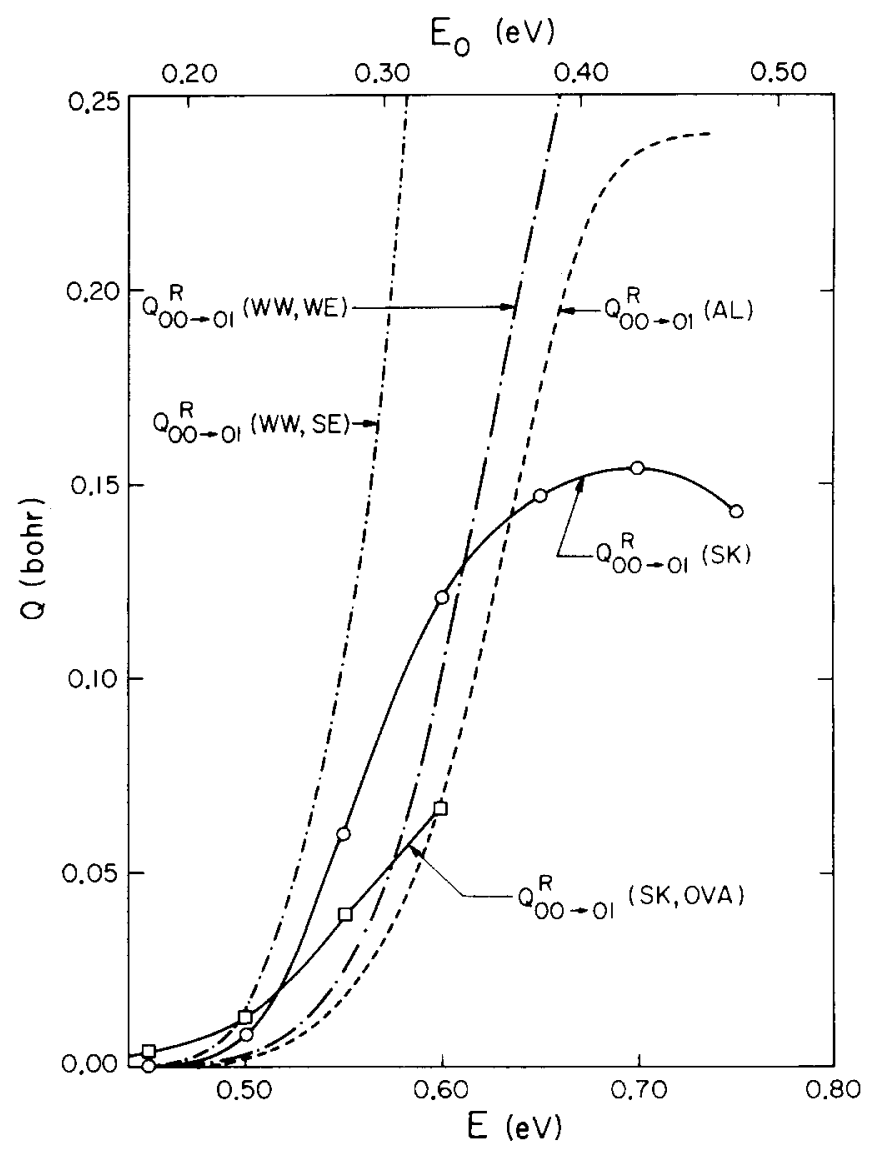

FIG. 23. Integral cross section $Q_{00 \rightarrow 01}^{R}$ vs total energy $E$ and translational energy $E_{0}$ for several exact and approximate coplanar calculations. $Q_{00-01}^{R}(\mathrm{SK})$ refers to the present converged results, $Q_{00-01}^{R}$ (SK, OVA) are the present one-vibration-approximation results, for a collinear $V_{\text {ref, }}$ obtained as described in Sec. II.D, $Q_{00 \rightarrow 01}^{R}(W W, W E)$ and $Q_{00-01}^{R}(W W, S E)$ are the results of distorted wave calculations of Ref. 15, and $Q_{00 \rightarrow 01}^{R}(\mathrm{AL})$ is the OVA result (on a different potential surface and using a method somewhat different from ours) of Ref. 10 。

ner analogous to that done for the converged cross sections in Fig. 21. Figure 22 indicates that the OVA rotational distributions fit the temperaturelike distribution given in Eq. (3.3) to about the same accuracy as the converged results. However, the OVA temperature parameters are somewhat higher (450-620 K), indicating that this approximate procedure predicts rotational distributions which are much broader than the converged ones.

\section{F. Comparisons with other coplanar calculations}

In Fig. 23 we plot our converged $Q_{00 \rightarrow 01}^{R}(\mathrm{SK})$ and the corresponding one-vibration-approximation results $Q_{00 \rightarrow 01}^{R}$ (SK, OVA) using a collinear reference function (see Sec. II. D), along with the results of two other studies on coplanar $\mathrm{H}+\mathrm{H}_{2} \cdot Q_{00 \rightarrow 01}^{R}(\mathrm{WW}, \mathrm{SE})$ and $Q_{00 \rightarrow 01}^{R}$ (WW, WE) come from two different applications of the distorted wave approximation by Walker and Wyatt ${ }^{15}$ on the Porter-Karplus surface. SE and WE refer, respectively, to the strong and weak expansion path choices of the nonreactive reference potential used to generate the distorted wavefunctions. $Q_{00 \rightarrow 01}^{R}(\mathrm{AL})$ is the coupled-channel result (using one variation of the OVA) 

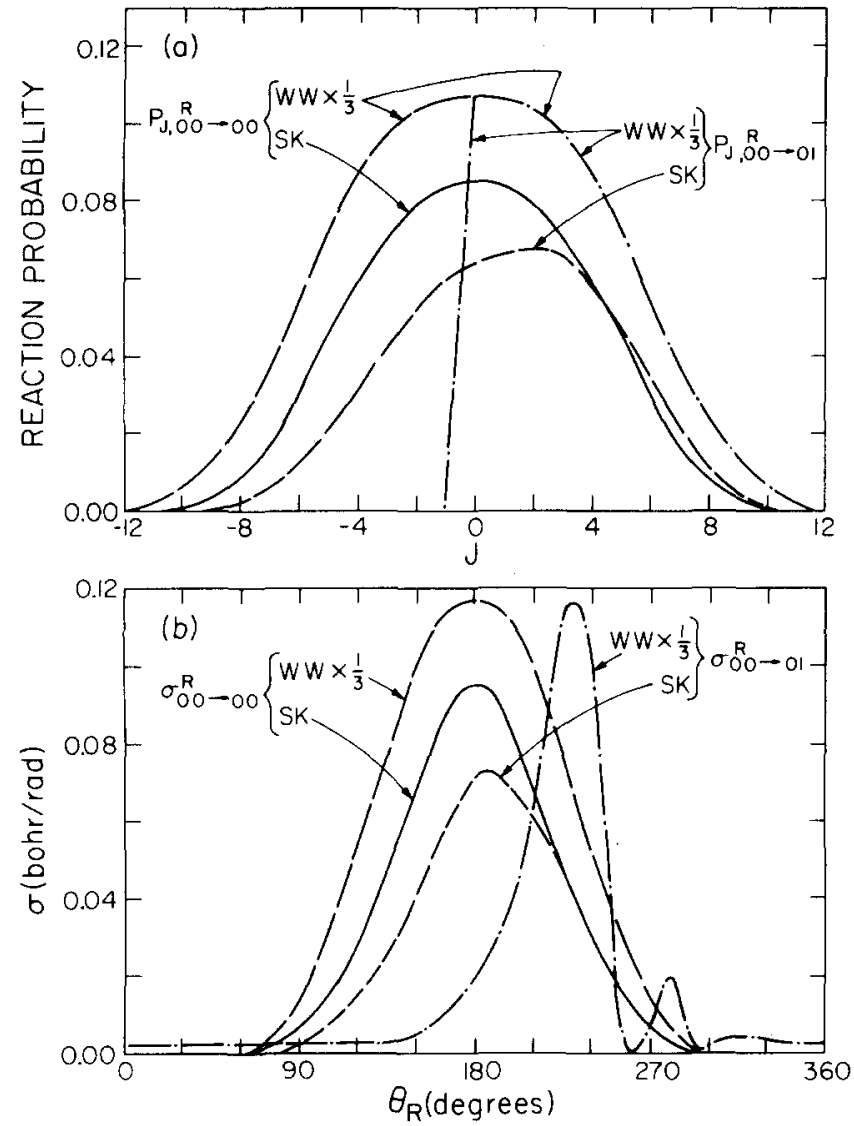

FIG. 24. Reaction probabilities $P_{J, 00 \rightarrow 00}^{R}$ and $P_{J, 00 \rightarrow 01}^{R}$ and differential cross sections $\sigma_{00 \rightarrow 00}^{R}$ and $\sigma_{00 \rightarrow 01}^{R}$ as a function of reactive scattering angle $\theta_{R}$. WW refers to the SE results of Ref. 15 with Iong dashed curves referring to $00 \rightarrow 00$ transitions and dash-dot curves to $00 \rightarrow 01$ ones. SK refers to the results of this paper (Figs. 2, 3, 8, and 9) with solid curves for $00 \rightarrow 00$ and short dashed for $00 \rightarrow 01$ transitions. WW results are at $E_{0}=0.34 \mathrm{eV}(E=0.61 \mathrm{eV})$, while $\mathrm{SK}$ results are at $E_{0}=0.33 \mathrm{eV}$ $(E=0.60 \mathrm{eV})$.

of Altenberger-Siczek and Light, ${ }^{10}$ in which an earlier calculation of Saxon and Light $t^{8}$ is corrected. These calculations were done for an analytical surface fitted to the $a b$ initio $\mathrm{SSMK}^{38}$ surface. The two approximate calculations of Walker and Wyatt seem to bracket our result at low energies, but for $E>0.60 \mathrm{eV}$, the absence of conservation of flux in the distorted wave calculation results in a gross overestimation of the integral cross sections. Thus, the distorted wave method remains accurate only when the reaction probabilities or cross sections are small. A similar conclusion was also drawn from an analogous collinear comparison. ${ }^{39}$. The shapes of the distorted wave differential cross section $\sigma_{00-00}^{R}$ vs reactive scattering angle $\theta_{R}$ (Fig. 24) are in good agreement with the corresponding results of our converged calculations, but not the magnitude of those cross sections. A similar comparison of the cross sections $\sigma_{00 \rightarrow 01}^{R}$ in that figure indicates serious disagreement in both shape and magnitude, apparently due to a much more rapid falloff in the distorted wave reaction probabilities with decreasing $J(J<0)$ than is the case with our results [as seen in Fig. 24(a)].

The results of Altenberger-Siczek and Light ${ }^{10}$ of Fig。
23 cannot be quantitatively compared with ours because of the difference in potential energy surfaces used in the two calculations, but some qualitative observations are nevertheless appropriate. First, the effective threshold energies (defined in Sec。III.C) of the integral cross sections are about $0.50 \mathrm{eV}$ for the converged $Q_{00 \rightarrow 01}^{R}(\mathrm{SK})$, $0.49 \mathrm{eV}$ for the $Q_{00-01}^{R}(\mathrm{SK}, \mathrm{OVA})$, and $0.53 \mathrm{eV}$ for $Q_{00+01}^{R}(\mathrm{AL})$. The difference of $0.04 \mathrm{eV}$ between these last two numbers is approximately equal to the 0.03 $\mathrm{eV}$ difference between the heights of the respective potential barriers $(0.396 \mathrm{eV}$ and $0.425 \mathrm{eV})$ in the surfaces used in the calculations. Since the properties of the saddle point regions of these surfaces are similar, one might expect that a small change in barrier height should indeed result in a correspondingly small change in effective threshold energy as observed. Second, even if the AL curve is shifted to lower energies by 0.03 $\mathrm{eV}$ to correct for this difference in barrier heights, it agrees neither with our converged nor with our OVA results; above the phenomenological threshold, it increases more rapidly with energy and to larger values than either of the latter. This may be due to differences in the characteristics of the two surfaces other than the barrier heights. Third, except for some possibly spurious oscillations, the Altenberger-Siczek and Light angular distributions (Figs. 8-10 of Ref. 10) have shapes which are generally similar to ours (Figs. 8, 9 of this paper) for all transitions considered. The dominant peak near $180^{\circ}$ in their angular distributions is somewhat narrower than ours and their reaction probabilities fall off more rapidly with increasing $|J|$ than do ours in Figs. 1-3. Both of these differences could be a result of the different potential surfaces used, since as seen in Figs. 4 and 10 the OVA does not strongly affect the shapes of $\sigma^{R}$ vs $\theta_{R}$ and $P^{R}$ vs $J$ curves.

\section{G. Rate constants}

In this section we examine the behavior of the parato-ortho thermal rate constant $k_{p-0}(T)$. The ortho-topara rate constant can, of course, be obtained from $k_{p \rightarrow 0}$ by using the easily calculable equilibrium constant for this reaction, ${ }^{40}$ computed for the coplanar world of this paper. To obtain $k_{p-0}(T)$ we first require the para-to-ortho cross sections $\bar{Q}_{v f}^{A}$ [summed over all final ortho states and averaged over initial (para) rotational polarizations]. These are listed in Table IV for both the converged and OVA calculations.

The planar para-to-ortho rate constant is given by

$$
k_{\mathrm{p} \rightarrow 0}(T)=\frac{1}{Z} \sum_{v=0}^{\infty} \sum_{\substack{j=0 \\(j=\text { ver })}}^{\infty}\left(2-\delta_{j 0}\right) e^{-E_{v f} / k T} k_{v j}^{A}(T),
$$

where $Z$ is the planar partition function

$$
Z=\sum_{v=0}^{\infty} \sum_{\substack{j=0 \\(j=e \text { eren })}}^{\infty}\left(2-\delta_{j 0}\right) e^{-E_{v j} / k T}
$$

and

$k_{v j}^{A}(T)=\left(\frac{2}{\mu}\right)^{1 / 2} \frac{1}{k T} \int_{0}^{\infty} e^{-E_{v j}^{\prime} / k T} \bar{Q}_{v j}^{A}\left(E_{v j}^{\prime}\right) E_{v j}^{\prime 1 / 2} d E_{v f}^{\prime}$. 
TABLE IV. Para-to-ortho integral cross sections (in bohr) for $\mathrm{H}+\mathrm{H}_{2} \bullet^{\mathrm{a}}$

\begin{tabular}{llll|lll}
\hline \hline & & \multicolumn{3}{c|}{ Converged } & \multicolumn{3}{|c}{ OVA } \\
$E$ & $\bar{Q}_{00}^{A}$ & $\bar{Q}_{02}^{A}$ & $\bar{Q}_{04}^{A}$ & $\bar{Q}_{00}^{A}$ & $\bar{Q}_{02}^{A}$ & $\bar{Q}_{04}^{A}$ \\
\hline 0.30 & $0.356(-11)$ & $\cdots$ & $\cdots$ & $0.543(-8)$ & $\cdots$ & $\cdots$ \\
0.35 & $0.144(-6)$ & $0.137(-7)$ & $\cdots$ & $0.343(-3)$ & $0.221(-4)$ & $\cdots$ \\
0.40 & $0.499(-4)$ & $0.868(-5)$ & $0.400(-4)$ & $0.280(-2)$ & $0.976(-3)$ & $0.228(-7)$ \\
0.45 & $0.299(-2)$ & $0.721(-3)$ & $0.104(-5)$ & 0.0249 & $0.958(-2)$ & $0.288(-3)$ \\
0.50 & 0.0543 & 0.0319 & $0.127(-3)$ & 0.0918 & 0.0476 & $0.336(-2)$ \\
0.55 & 0.391 & 0.140 & $0.309(-2)$ & 0.294 & 0.166 & 0.0250 \\
0.60 & 0.841 & 0.361 & 0.0233 & 0.533 & 0.339 & 0.0727 \\
0.65 & 1.025 & 0.619 & 0.0724 & $\mathrm{~b}$ & $\mathrm{~b}$ & $\mathrm{~b}$ \\
0.70 & 1.134 & 0.877 & 0.194 & $\mathrm{~b}$ & $\mathrm{~b}$ & $\mathrm{~b}$ \\
0.75 & 1.204 & 1.088 & 0.344 & $\mathrm{~b}$ & $\mathrm{~b}$ & $\mathrm{~b}$ \\
\hline \hline
\end{tabular}

${ }^{a}$ All cross sections have been summed over final ortho (odd $j^{\prime}$ ) states and averaged over initial rotational polarizations. The numbers in parentheses indicate powers of 10 by which the numbers preceding them are multiplied.

${ }^{\mathrm{b}}$ These cross sections were not calculated, but estimates of their values (through various extrapolation procedures) were used in the rate constant calculations. Because they involve higher energy results, their contributions to the rate constants are small, and the errors in these rate constants resulting from the extrapolations are usually less than $10 \%$.

$E_{v f}$ is the vibration-rotation energy of the initial state with quantum numbers $v j$, and $E_{v j}^{\prime}$ is the translational energy relative to that state $\left(E_{v f}^{\prime}=E-E_{v j}\right) . \mu$ is the reduced mass associated with the relative motion of the reagents and the factor $2-\delta_{j 0}$ is introduced to account explicitly for rotational degeneracy. The initial spin degeneracy is 1 for all para states. The rate constant thus defined has the units $\mathrm{cm}^{2} /$ molecule. $\mathrm{sec}$, which is appropriate for a planar world in which concentrations are measured in molecule $/ \mathrm{cm}^{2}$.

Using Eqs. (3.4)-(3.6) along with the data in Table IV, $k_{\mathfrak{p} \rightarrow \mathrm{o}}(T)$ has been calculated, and the resulting Arrhenius plots for the vibrationally converged and OVA $\left[V_{\text {ref }}=V\left(r_{\lambda}, R_{\lambda}, \gamma_{\lambda}=0\right)\right]$ results are presented in Fig. 25. As might be expected from the appearance of the integral cross sections in Fig. 16, the OVA rate constant is considerably larger than the converged one at low temperatures, with the ratio of the two being 12.4 and 2.83 at $200 \mathrm{~K}$ and $300 \mathrm{~K}$, respectively. At high temperatures, the two rate constants approach each other quite closely, a reflection of the similar effective threshold energies of the converged and OVA cross sections. The high temperature portions of the Arrhenius plots in Fig. 25 are nearly linear with resulting Arrhenius activation energies of 5.2 and $5.0 \mathrm{kcal} / \mathrm{mole}$ for the converged and OVA results, respectively. The high temperature Arrhenius straight line corresponding to the converged results is represented by the dashed line of Fig. 25. We will defer a detailed comparison of these rate constants with those of accurate one- and three-dimensional calculations and with approximate theoretical and experimental ones to a separate paper.

\section{CONCLUSION}

It should be apparent from the wealth of dynamical information presented in Sec. III that these calculations can be extremely useful to our understanding of chemical dynamics. We would like to stress that these calculations are not overly time consuming, but it is un- likely that similar ones will be done on more than the very simplest of chemical systems. Rather, the primary emphasis is to use these results as benchmarks against which approximate theories may be compared, with the hope that these theories may be in turn applied

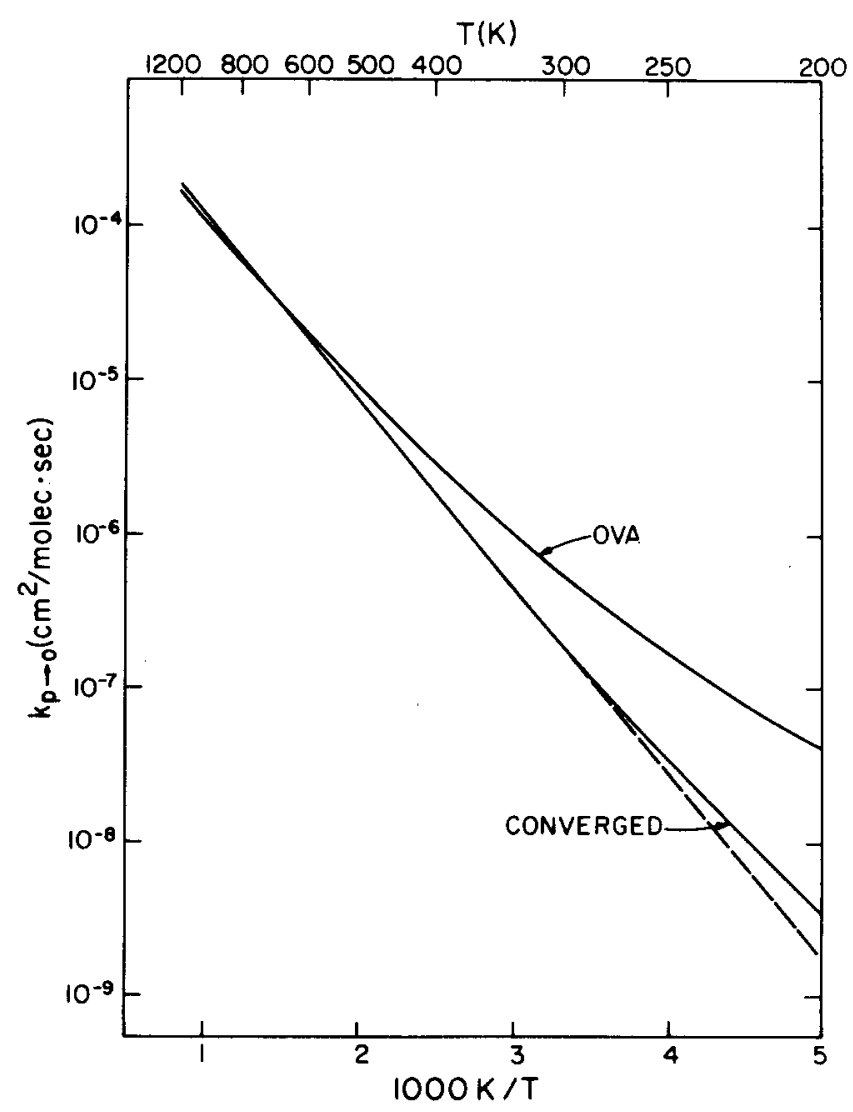

FIG. 25. Arrhenius plot of the converged and OVA para-toortho coplanar thermal rate constants for $\mathrm{H}+\mathrm{H}_{2}$ for the converged and OVA (collinear reference potential) results. The dashed straight line is tangent to the converged one at high temperatures. 
to more complicated systems. The comparisons with approximate calculations presented in Sec. III. F were incomplete in that the results of quasiclassical and semiclassical coplanar calculations on the PorterKarplus $\mathrm{H}_{3}$ surface are needed to assess the quantitative accuracy of these important approximate theories. Also requiring further consideration is the use of collinear-type theories to provide approximate coplanar results, and similarly of coplanar theories to describe the three-dimensional world。 ${ }^{41}$ This was discussed briefly in Sec. III. D and will be further investigated in a separate paper.

The coplanar calculations are also important in elucidating what kinds of phenomena are significant in chemical reactions. The magnitude of the quantum symmetry oscillations in the para-to-para angular distributions (Sec, III. B) as a function of energy (Sec。 III. C) and their absence in the corresponding integral cross sections are a good example. Such quantum symmetry effects may eventually be a useful experimental tool for characterizing reactive potential surfaces. Also of great importance is the characterization of the reaction in terms of direct and resonant mechanisms. This was briefly mentioned in Sec。II. B, where we remarked that the reaction appeared to be completely dominated by the direct mechanism. A more detailed analysis at energies higher than were considered in this work indicates that in the neighborhood of certain energies (such as $E \sim 0.92 \mathrm{eV}$ ) this no longer seems to be correct as very significant resonantlike effects are observed. The importance of these resonant processes is discussed elsewhere. ${ }^{32}$

Finally, as was mentioned in the introduction, these calculations are significant in that they demonstrate the feasibility of the method outlined in Paper I for doing quantum 2D scattering calculations. Extension of this method to the 3D problem has recently been accomplished, ${ }^{12,21}$ and the results of these $3 \mathrm{D}$ calculations and their comparison with $2 \mathrm{D}$ and $1 \mathrm{D}$ ones should be extremely useful to our understanding of chemical dynamics.

\section{ACKNOWLEDGMENT}

Special thanks are due to Professor J. D. Roberts, who as chairman of the Division of Chemistry and Chemical Engineering at Caltech during the crucial stages of this research mustered the financial resources and offered the encouragement which were central to its successful completion. We thank Ambassador College for generous use of their computational facilities, We also thank Professor Donald G. Truhlar for useful comments.

${ }^{*}$ Work supported in part by the United States Air Force Office of Scientific Research (Grant No. AFOSR-73-2539).

fWork performed in partial fulfillment of the requirements for the Ph.D. degree in Chemistry at the California Institute of Technology.

\$Contribution No. 5249 .
${ }^{1}$ M. Karplus, R. N. Porter, and R. D. Sharma, J. Chem. Phys. 43, 3259 (1965); the classical angular distribution is reported in Ref. 9 .

${ }^{2}$ R. P. Saxon and J. C. Light, J. Chem. Phys. 57, 2758 (1972)。

${ }^{3}$ J. M. Bowman and A. Kuppermann, J. Chem. Phys. 59, 6524 (1973).

${ }^{4} J$. D. Doll, T. F. George, and W. H. Miller, J. Chem. Phys. 58,1343 (1973).

${ }^{5}$ J. J. Tyson, R. P. Saxon, and J. C. Light, J. Chem. Phys. 59, 363 (1973).

${ }^{6}$ D. G. Truhlar and A. Kuppermann, J. Chem. Phys. 56, 2232 (1972).

${ }^{7}$ G. C. Schatz and A. Kuppermann, J. Chem. Phys. 59, 964 (1973).

${ }^{8}$ R. P. Saxon and J. C. Light, J. Chem. Phys. 56, 3874 (1972); 56,3885 (1972).

${ }^{9}$ G. Wolken and M. Karplus, J. Chem. Phys. 60, 351 (1974).

${ }^{10} \mathrm{~A}$. Altenberger-Siczek and J. C. Light, J. Chem. Phys. 61, 4373 (1974).

${ }^{11}$ A. Kuppermann, G. C. Schatz, and M. Baer, J. Chem. Phys. 61, 4362 (1974).

${ }^{12}$ A. Kuppermann and G. C. Schatz, J. Chem. Phys. 62, 2502 (1975).

${ }^{13}$ K. T. Tang and M. Karplus, Phys. Rev. A 4, 1844 (1971).

${ }^{14} \mathrm{~K}$. T. Tang and B. H. Choi, J. Chem. Phys. 62,3642 (1975).

${ }^{15}$ R. B. Walker and R. E. Wyatt, J. Chem. Phys. 61,4839 (1974).

${ }^{16}$ Reference 2 contains an extensive list of collinear studies of this reaction.

${ }^{17} \mathrm{H}$. S. Johnston, Gas-Phase Reaction Rate Theory (Ronald, New York, 1966), Chap. 10 and references therein.

${ }^{18} \mathrm{~A}$. Kuppermann, G. C. Schatz, and M. Baer, J. Chem. Phys. 65, 4596 (1976), preceding paper, referred to as Paper I in the present paper.

${ }^{19}$ R. N. Porter and M. Karplus, J. Chem. Phys. 40, 1105 (1964)。

${ }^{20}$ We prefer the terms "coupled channel" or "coupled equation" rather than "close coupling" because we have found that many channels are needed to achieve convergence, including those which are not "close" to either the initial or final states considered.

${ }^{21} \mathrm{G}$. C. Schatz and A. Kuppermann, J. Chem. Phys. 65, 4642 (1976); 65, 4668 (1976), following two papers.

${ }^{22}$ Double precision (64 bit) arithmetic was used for all calculations.

${ }^{23} \mathrm{As} J$ increases, the repulsive centrifugal potential increases, effectively raising the potential in the interaction region and decreasing the reaction probability. The "physical" scattering wavefunction becomes small in the interaction region as this happens. On the other hand, when integrating the coupled-channel equations, the closed-channel wavefunctions always increase exponentially in the direction of integration because of the dominance of the increasing exponential solution over the decreasing one. For large $J$, all channels are closed in the strong interaction region. As a result, the solutions generated by propagation into that region (the plus solutions) become exponentially large, whereas those generated by integrating in the outward direction (the minus solutions) are small in the strong interaction region when compared with the values they attain outside of it. These minus solutions, which decrease exponentially as one penetrates the potential barrier, are the only physically meaningful ones and therefore the only ones which will contribute significantly to the physical solution. This implies that the matching procedure will attach a very small weight to the plus solutions [i.e., $\mathbf{C}_{J}^{+}\left(\mathbf{C}_{J}^{-}\right)^{-1} \cong \mathbf{O}$ in Eq. $(4,44)$ of Paper I]。 From Eqs. (5.9) $-(5.14)$ and especially $(5.18)$ of Paper I, we see that $c_{J}^{+}\left(c_{j}^{-}\right)^{-1}=0$ leads to a reactance matrix which is independent of any information about the matching, and hence the resulting nonreactive transition probabilities will not be 
sensitive to the lack of orthogonality of $\mathbf{S}_{\nu \lambda}^{J}$.

${ }^{24}$ For example, see R. Courant and D. Hilbert, Methods of Mathematical Physics (Interscience, New York, 1970), Vol. I, p. 4.

${ }^{25}$ We have tested both expressions in doing the OVA calculations, and have found that Eq. $(3.48)$ of Paper I leads to a much poorer approximation to the converged results than does Eq. (2.10) of the present paper, and that use of the former expression can actually lead to spurious resonantlike behavior in some transition probabilities. This could presumably result from the fact that in Eq. (2.10), we have analytically assumed completeness in carrying out the matrix multiplication present in Eq. (3.48) of Paper I, so the effects of a severe basis set truncation might be less important than in Eq. (3.48) of $I$, where this completeness is neither assumed nor explicitly included.

${ }^{26} \mathrm{~J}$. Geddes, H. F. Krause, and W. L. Fite, J. Chem. Phys. 56,3298 (1972).

${ }^{27}$ G. H. Kwei, V. W. S. Lo, and E. A. Entemann, J. Chem. Phys. 59, 3421 (1973).

${ }^{28}$ D. A. Micha, Phys. Rev. 162, 88 (1967); D. A. Micha, Chem. Phys. Lett. 1, 139 (1967); R. D. Levine, B. R. Johnson, J. T. Muckerman, and R. B. Bernstein, J. Chem. Phys. 49, 56 (1968); R. D. Levine and R. B. Bernstein, J. Chem. Phys. 53, 686 (1970).

${ }^{29}$ This argument does not rule out vibrational internal excitation resonances. These resonances have actually been observed in planar and $3 \mathrm{D} \mathrm{H}+\mathrm{H}_{2}{ }^{32}$ (at higher energies than are considered in this paper). As one might expect, however, they affect more than one partial wave ${ }^{32}$ and are probably not responsible for the oscillations observed here.

${ }^{30}$ For examples, see J. M. Farrer and Y. T. Lee, J. Chem. Phys. 56, 5801 (1972); P. E. Siska, J. M. Parson, T. P. Schafer, and Y. T. Lee, J. Chem. Phys. 55, 5762 (1971),
J. M. Farrar and Y。T. Lee, J. Chem. Phys. 57, 5492 (1972); H. Haberland, C. H. Chen, and Y. T. Lee, At. Phys. 3, 339 (1973); B. Andresen and A. Kuppermann, Mol. Phys. 30,997 (1975).

${ }^{31}$ J. M. Bowman, A. Kuppermann, J. T. Adams, and D. G. Truhlar, Chem. Phys. Lett. 20, 229 (1973).

${ }^{32}$ G. C. Schatz and A. Kuppermann, Phys. Rev. Lett. 35, 1266 (1975).

${ }^{33}$ (a) D. J. Diestler, J. Chem. Phys. 54, 4547 (1971); (b) J. W. Duff and D. G. Truhlar, Chem. Phys, Lett. 23, 327 (1973).

${ }^{34}$ A. B. Elkowitz and R. E. Wyatt, J. Chem. Phys. 63, 702 (1975).

${ }^{35}$ R. A. Marcus, J. Chem. Phys. 46, 959 (1967).

${ }^{36}$ Using microscopic reversibility the dependence of $\bar{Q}_{0 j \rightarrow 00}^{R}$ on $j$ can be extracted from the $\bar{Q}_{00 \rightarrow 0 j}^{R}$ in Fig. 21 by multiplying the latter by the ratio $2 k_{00} /\left[k_{02}\left(1+\delta_{j 0}\right)\right]$, where the wave numbers $k_{00}$ and $k_{02}$ refer to the $v=0, j=0$ and $v=0, j=2$ states of $\mathrm{H}_{2}$, respectively, and $\left(1+\delta_{j 0}\right) / 2$ is a degeneracy factor.

${ }^{37}$ The distribution may be derived from an information theoretic formalism [see, for example, A. Ben-Shaul, R. D. Levine, and R. B. Bernstein, J. Chem. Phys. 57, 5427 (1972)] by assuming that the surprisal function is linear in the product rotational energy. Note that the $2 \mathrm{D}$ translational density of states is independent of $E_{j^{\prime}}$ and is therefore omitted from Eq. (3.3).

${ }^{38}$ I. Shavitt, R. M. Stevens, F. L. Minn, and M. Karplus, J. Chem. Phys. 48, 2700 (1968).

${ }^{39}$ R. B. Walker and R. E. Wyatt, Chem. Phys. Lett. 16, 52 (1972).

${ }^{40}$ A. Farkas, Orthohydrogen, Parahydrogen and Heavy Hydrogen, (Cambridge U.P., London, 1935), p. 13.

${ }^{41}$ For a recent paper on this subject, see R. B. Walker and R. E. Wyatt, Mol. Phys. 28, 101 (1974). 